\title{
A Survey on Infrastructure-Based Vehicular Networks
}

\author{
Cristiano M. Silva, ${ }^{1}$ Barbara M. Masini, ${ }^{2}$ Gianluigi Ferrari, ${ }^{3}$ and Ilaria Thibault ${ }^{4}$ \\ ${ }^{1}$ Departamento de Tecnologia, Universidade Federal de São João del-Rei, São João del-Rei, MG, Brazil \\ ${ }^{2}$ National Research Council, Institute of Electronics, Computer and Telecommunication Engineering (CNR-IEIIT), Bologna, Italy \\ ${ }^{3}$ IoT Lab, Department of Information Engineering and DISS-Road Security Center, University of Parma, Parma, Italy \\ ${ }^{4}$ Vodafone Group Research and Development, Newbury, UK
}

Correspondence should be addressed to Cristiano M. Silva; cristiano@ufsj.edu.br

Received 23 December 2016; Revised 17 April 2017; Accepted 23 April 2017; Published 6 August 2017

Academic Editor: Dik Lun Lee

Copyright (C) 2017 Cristiano M. Silva et al. This is an open access article distributed under the Creative Commons Attribution License, which permits unrestricted use, distribution, and reproduction in any medium, provided the original work is properly cited.

\begin{abstract}
The infrastructure of vehicular networks plays a major role in realizing the full potential of vehicular communications. More and more vehicles are connected to the Internet and to each other, driving new technological transformations in a multidisciplinary way. Researchers in automotive/telecom industries and academia are joining their effort to provide their visions and solutions to increasingly complex transportation systems, also envisioning a myriad of applications to improve the driving experience and the mobility. These trends pose significant challenges to the communication systems: low latency, higher throughput, and increased reliability have to be granted by the wireless access technologies and by a suitable (possibly dedicated) infrastructure. This paper presents an in-depth survey of more than ten years of research on infrastructures, wireless access technologies and techniques, and deployment that make vehicular connectivity available. In addition, we identify the limitations of present technologies and infrastructures and the challenges associated with such infrastructure-based vehicular communications, also highlighting potential solutions.
\end{abstract}

\section{Introduction}

More and more vehicles are connected to the Internet through vehicle-to-anything (V2X) communication technologies, changing the automotive industry and the transportation system. By embedding computing devices into cars, roads, streets, and transit equipment (such as street signs, radars, traffic cameras, and others), we will be capable of digitalizing the transportation system [1], letting vehicles autonomously exchange data with other vehicles (V2V communications), with the network infrastructure (V2I communications), with the road infrastructure (V2R communications), with pedestrians (V2P communications), and so forth. Data collected by vehicles and pushed back from a remote control center will contribute to developing new services for vehicular users, automotive industries, and network providers. Connectivity is, then, the key issue for the provision of value-added services ranging from road safety, traffic management, and environment monitoring, up to autonomous driving. Connectivity will also enhance data collection and data exchange, driving new social and economic models which will impact the worldwide society and business.

As indicated by the European Transportation Policy [2], the use of Intelligent Transportation Systems is one of the key technologies for improving the safety, efficiency, and environmental friendliness of the transport industry. Intelligent Transportation Systems are grounded on sophisticated communication networks receiving data from several entities composing the traffic system. Data is processed and translated into useful information and recommendations to assist users of the transportation system and transit authorities. Such sophisticated communication network is commonly referred to as vehicular network [3-5]. Vehicular networks connect vehicles to provide a platform for the future deployment of large-scale and highly mobile applications. Applications are endless: driver assistance for faster, less congested, and safer roads; more efficient use of the transportation system; more efficient planning of routes and control of the traffic flow; more secure and greener traffic through digital driver 
assistance; better planning and evolution of the system as a whole due to the availability of historical data, based on traffic and utilization trends detected via data mining techniques and autonomous driving.

The broad range of applications can be enabled by two main kinds of connectivity: infrastructure-based communications (hereafter V2I) and direct communications between vehicles (hereafter V2V). V2V communications are essentials for beaconing; coverage extensions; and very low latency applications. Focusing on V2I communications, vehicles may communicate to roadside units (RSUs) through short range communications or even to a remote control center by exploiting wide area networks. The infrastructure plays a coordination role by gathering global or local (potentially real time) information and then "suggesting" appropriate behaviors to drivers or managing specific services. These applications typically rely on an extended coverage, such as data collection at a remote infrastructure for traffic management, environmental monitoring, smart navigation, smart logistic, predictive vehicles maintenance, and pay as you drive.

Transit authorities may also deploy a dedicated infrastructure for vehicular communications. Such dedicated infrastructure is assumed to be reliable and trusted, enabling transit authorities to collect data from several sensors of the vehicle (airbag actioning, braking, videos, etc.), providing data for the real-time programming of smart traffic lights, traffic warnings, routing of emergency vehicles, road signing, and even autonomous driving in the near future. On the other hand, V2V communications are typically devoted to improve safety, providing low latency, fast network connectivity, and highly secure and high-speed communication typically used in platooning and collision avoidance systems [6-8].

Besides being needed for next-generation mobile applications, the use of a dedicated infrastructure for the vehicular communication also provides clear benefits in creating shortcuts in the graph of connections, restricting the ad hoc communication to small regions and position-based applications. At the end of 2016, Audi showed, for example, the first commercial V2I communication system in the United States: car-to-traffic-light chats to know how long the red light lasts. In the near future, such systems might help to save fuel and cut pollution or to provide infotainment and commercial information [9]. Whenever messages have to travel long distances, they can be tunneled, via the communication infrastructure, to the target region, improving network connectivity [10-15]. On the other hand, the deployment of a large-scale infrastructure is likely to demand huge investments. Hence, the research community has turned its attention to strategies for efficient deployment of a distributed communication infrastructure.

In this context, standardization entities are moving to develop reliable and secure wireless communications specifications to enable truly interoperable services worldwide. Allocation of dedicated spectrum for V2X communications both in US and in Europe has triggered standardization efforts in both regions to address a wide variety of V2X scenarios. As a result, two families of standards have been completed: the IEEE WAVE, with the 802.11p as the physical and lower-MAC layer standard, in 2010 in the US, and the first release of the ETSI intelligent transport systems (ITS), denoted as ETSI ITS-G5, in 2013 in Europe. In early 2014, different working groups within 3GPP have also started studying V2X as an additional feature for LTE-Advanced [16-18] and as a native feature for 5G. Other alternative solutions may be based on the rapidly spreading low-power wide area networks (LPWANs), which exploit sub-gigahertz unlicensed frequency bands and allow long-range radio links [19]. Owing to the long transmission range, sub-gigahertz technologies are attractive to support low data rate and longlasting communications in vehicular networks.

This article is organized as follows. Section 2 briefly overviews the research in infrastructure-based vehicular networks from 2003 to 2016 . Section 3 refers to vehicle-toinfrastructure (V2I) architectures. Section 4 refers to wireless access technologies and communication techniques. Section 5 discusses the deployment of infrastructure for vehicular networks. Section 6 concludes the article and points out to future challenges sketching potential solutions.

\section{Overview of the Research in Infrastructure-Based Vehicular Networks}

In the context of vehicular networks, infrastructure is a set of specialized communication devices supporting the network operation. Common properties include (but are not restricted to) network centrality, communication bandwidth, storage space, and high availability. Because vehicular network devices are initially envisioned to be located at roadsides, they are commonly referred to as RSUs and may provide a large number of functions, such as the following:

(i) Broadcast [20]

(ii) Channel allocation [21]

(iii) Caching [22]

(iv) Content download [23, 24]

(v) Data dissemination [25]

(vi) Data aggregation [26]

(vii) Data scheduling [27]

(viii) Gaming \& streaming [28, 29]

(ix) Gateway [11, 22, 30]

(x) Hand-off [31-33]

(xi) Vehicles localization [34, 35]

(xii) QoS [36-39]

(xiii) Real-time support [40-42]

(xiv) Routing [14, 43, 44]

(xv) Security [45-48]

(xvi) Multihop comm [49].

Several technologies may be embedded in RSUs. Banerjee et al. [12] present an in-depth discussion and comparison of such technologies. Although most of the works consider stationary infrastructure, several papers [44, 50-55] propose 
mobile architectures (public transportation buses, cabs, and ordinary vehicles). There are also proposals considering the use of low cost devices as an infrastructure [56], while other proposals consider the use of external communication devices (such as public Wi-Fi) [57].

The following basic groups can be envisioned to categorize the studies dealing with infrastructure-based vehicular networks.

(i) Architectures for infrastructure-based vehicular networks (Section 3): works are proposing new architectures, testbeds, proofs of concept, field trials and experimentations, and hardware studies.

(ii) Communication in infrastructure-based vehicular networks (Section 4): works are studying the vehicular communication in terms of protocols, data dissemination strategies, routing, connectivity, low level aspects of the communication, channel allocation, hand-off strategies, network throughput, quality of service, real-time messaging, and multihop data dissemination.

(iii) Security in infrastructure-based vehicular networks (Section 4.9): we present only a brief discussion of security in infrastructure-based vehicular networks (most of the strategies we could find target the ad hoc scenario).

(iv) Deployment of infrastructure for vehicular networks (Section 5): works are proposing strategies to physically locate the infrastructure, theoretical studies discussing metrics and strategies to evaluate deployments, and theoretical studies about requirements, features, or properties of network deployment.

In the early 2000s, most of the researches dealt with low level aspects of the communication. The high mobility of nodes, the constantly changing topology of vehicular networks, and the connectivity dependent on the location bring several interesting research challenges. The US Department of Transportation (USDOT) (US Department of Transportation, Research and Innovative Technology Administration, http://www.its.dot.gov/vii/) shows a clear focus on integrating vehicles for making the current transportation system more intelligent [58]. The research community is aware of the challenges imposed by vehicular communications. As we increase the number of participating vehicles in the network, the communication channel receives an increasing demand for multiple vehicles attempting to send and receive data simultaneously. The ASTM (American Society for Testing and Materials) and IEEE (Institute of Electrical and Electronics Engineers) adopt the Dedicated Short Range Communication (DSRC) (http://www.its.dot.gov/DSRC/) standard providing wireless communication capabilities for transportation applications within a $1,000 \mathrm{~m}$ range in highway speeds. DSRC provides seven channels in the spectrum between 5.850 and $5.925 \mathrm{GHz}$ licensed for Intelligent Transportation Systems applications (Intelligent Transportation Systems Radio Service, ITS-RS) with channels designated for different applications, plus one channel reserved for $\mathrm{V} 2 \mathrm{~V}$ communications [4].
In the next few paragraphs, we overview the research conducted in infrastructure-based vehicular networks from 2003 to 2016.

In 2003-2004, researchers focused on low level details (physical/MAC layers) of the vehicular communication. While [10] investigates the capacity of the wireless channel, the work [59] investigates channel access. Only few works start considering the impact of lower levels on applications, as, for example, in [60]. However, in these years, Intelligent Transportation Systems in general are considered; connected vehicles are included in the systems but with a lower and different attention.

In 2005-2006, most of the researches are still on evaluating low level aspects of the communication, but the focus is not only on V2I communications, but also on V2V. The research community also visualizes the possibility of offering Internet access to vehicles. We notice works investigating novel architectures [11,30], hand-off strategies [31-33], network throughput $[61,62]$, and multihop communication [63].

In 2007-2008, the research community turns its attention to the validity of mobility models employed in vehicular simulations. They start to exploit the possible usage of the infrastructure for vehicular networks in terms of security and applications. The research community demonstrates interest in the potential use of public $\mathrm{Wi}$-Fi access points to enhance the vehicular communication, and we notice the first works dealing with infrastructure deployment. We also find two surveys $[3,5]$ addressing vehicular ad hoc networks (VANETs). These works discuss vehicle-to-vehicle communication, the impact of decentralization, channel access, market issues, security, privacy, and validation of VANETs simulations. The infrastructure gains visibility as the research community realizes its importance to support the dissemination of data in vehicular networks $[25,64]$. In order to reduce the deployment costs, some researchers focus on alternative less-expensive methods to achieve the benefits of a dedicated infrastructure, such as using publicly Wi-Fi [57] and the adoption of virtual infrastructure [50,65] using vehicles.

The research community also realizes that bringing Internet access to drivers enables the development of a myriad of vehicular applications and traffic information systems. Examples of these envisioned applications are (i) Pothole Patrol [66] to monitor roads conditions; (ii) Waze Mobile App [67]; (iii) Peer on Wheels [68] to monitor traffic conditions; and (iv) RoadSpeak [69] to enable chatting between drivers. The performance of the network is deeply studied in works $[58,70-72]$, and the available communication hardware is evaluated in order to find out better solutions [12]. Testbeds are proposed in $[73,74]$ to evaluate practical aspects related to the vehicular communication, while requirements of privacy and security of the infrastructure are addressed in [47]. Moreover, Fiore and Härri [75] present an in-depth analysis of the topological properties of a vehicular network and found that simulation results are strongly affected by the mobility model, and they question the validity of studies conducted under unrealistic car mobility scenarios.

In $2009-2010$, over $40 \%$ of the works are addressing infrastructure deployment using heuristics [76-79], clusters [80], or proposing metrics [81]. A virtual infrastructure using 
buses is presented in [51], while a secure infrastructure is proposed in [46]. When considering vehicle-to-infrastructure communication, we notice works addressing the scheduling [27], real-time communication [40, 41], delivery in sparse networks [42], analysis of connectivity [82-84], broadcast protocols [20], and cooperative georouting [43].

In 2011-2012, we notice works addressing infrastructure deployment in terms of probabilistic models $[85,86]$, linear programming formulations [87-89], heuristics [90], genetic algorithms [91], and game-theory [92]. In terms of architecture, we notice analytic models for the selection of communicating devices $[88,93]$, proposals for light-weight infrastructures employing relay nodes [13], virtual infrastructures using the publish-subscribe paradigm [52], biologically inspired solutions [94], and reputation mechanisms [45]. In terms of communication, we notice works addressing routing [14, 44, 95], cooperative data dissemination [22], QoS controlled media access [36], multihop communication [49], content download [23], and data traffic [96, 97]. Mobility is addressed in [98-100].

In 2013-2014, we notice works addressing high-level aspects of the infrastructure-based communication. Tonguz and Viriyasitavat [53] propose a self-organizing network using cars as RSUs. Luan et al. [56] propose the use of roadside buffers and cheap devices for store-and-forward messages to passing vehicles. Sommer et al. [54] study signal attenuation by buildings and propose the use of parked cars to help the signal propagation. When we consider communication, Harigovindan et al. [21] develop a mechanism for fair channel allocation, and Bruno and Nurchis [26] propose a mechanism to eliminate redundancy in data collected by vehicles in a distributed basis. A comparison among the impacts of different infrastructures on vehicular traffic performance is proposed in [101]: here, both cellular networks, broadcasting technologies, and $\mathrm{V} 2 \mathrm{~V}$ communications are analyzed and their performance is investigated when small and frequent traffic information fare was gathered from vehicles and retransmitted back to vehicles. The impacts of the number and position of RSUs are addressed, giving some answers to the deployment of new infrastructures on the roadside.

Deployment of infrastructure for vehicular networks is also addressed employing several techniques, such as genetic approaches [102], Voronoi diagrams [103], analytic models [104], randomized algorithms [105], content download [24], bipartite graphs [106], and intersection priority [107]. In [108], a cross-network information dissemination (which anticipates a topic discussed in the next time slot), denoted as "Cross-Network Effective Traffic Alert Dissemination" (XNETAD), is proposed and experimentally validated: by leveraging the spontaneous formation of local Wi-Fi VANETs, with direct connections between neighboring vehicles, traffic alerts received from the cellular network are quickly disseminated.

In 2015-2016, vehicular networks in general acquire still more importance [109]: this is, for example, demonstrated by the number of papers which contain the keywords vehicular networks of IEEEXplore database: 2,462 conference publications, 1,319 journals and magazines, and 336 early access articles, of which 126 conferences and 52 journals are related to vehicle-to-infrastructure communications. Attention is devoted to high precision positioning $[110,111]$ for quality of applications enhancements and cooperative transmission [112-114] to improve channel allocation and resource management in network infrastructure such as base stations and relays. Heterogeneous vehicular networks are also considered [115], where heterogeneity may be in wireless access technologies [116], vertical handovers [117], architectures, and autonomous driving [118]. Attention is also devoted to information acquisition [119] (also with crowd sensing $[120,121])$ and to information dissemination [122-124]. The infrastructure is very often present for vehicular connectivity [125], but device-to-device (D2D) solutions gain an increasing importance $[18,126]$. The possible adoption of LTE not only through the infrastructure of eNodeBs but also in direct mode enables new potential applications, also with low latency. Communication faces the issue of beaconing for vehicular awareness, addressing both the problem of channel load and adaptive beaconing. The scientific community focuses on safety applications [127] for different environments: platooning, lane changing, collision avoidance, and so forth. There are also some proposals focusing on planning [128-130] and managing [37-39, 131] vehicular networks.

\section{Architectures of Infrastructure-Based Vehicular Networks}

A general architecture is shown in Figure 1: vehicles are equipped with connected on-board units (OBUs) which can transmit data to other vehicles or to a remote control center exploiting different communication technologies and different infrastructures (cellular infrastructure, roadside infrastructures based on short range communications, and others). Important projects start to show relevant results, such as Fleetnet [132], Berkeley's California PATH (http://www.path.berkeley.edu/), and CarTel [133]. In particular, CarTel evaluates the V2I communication with city-wide trials in Boston and reports the upload bandwidth to vehicles using the unplanned open residential access. One of the main conclusions of CarTel is that the plethora of $802.11 \mathrm{~b}$ access points spreading in cities can provide intermittent connectivity with high performance while available. Moreover, Wu et al. [30] state that infrastructure assessments are necessary to (i) evaluate communications architectures to identify those best suited for providing high bandwidth communications to travelers; (ii) examine design options and trade-offs; and (iii) quantitatively assess alternate approaches and evaluate their performance and reliability under realistic vehicle traffic conditions.

We identity the following categories of works addressing architectures for vehicular networks:

(i) Analytic Studies Addressing Specific Aspects of the Infrastructure

(ii) Benefits of Incorporating the Infrastructure in Vehicular Networks

(iii) Cooperative Architectures

(iv) Light and Smart Architectures 


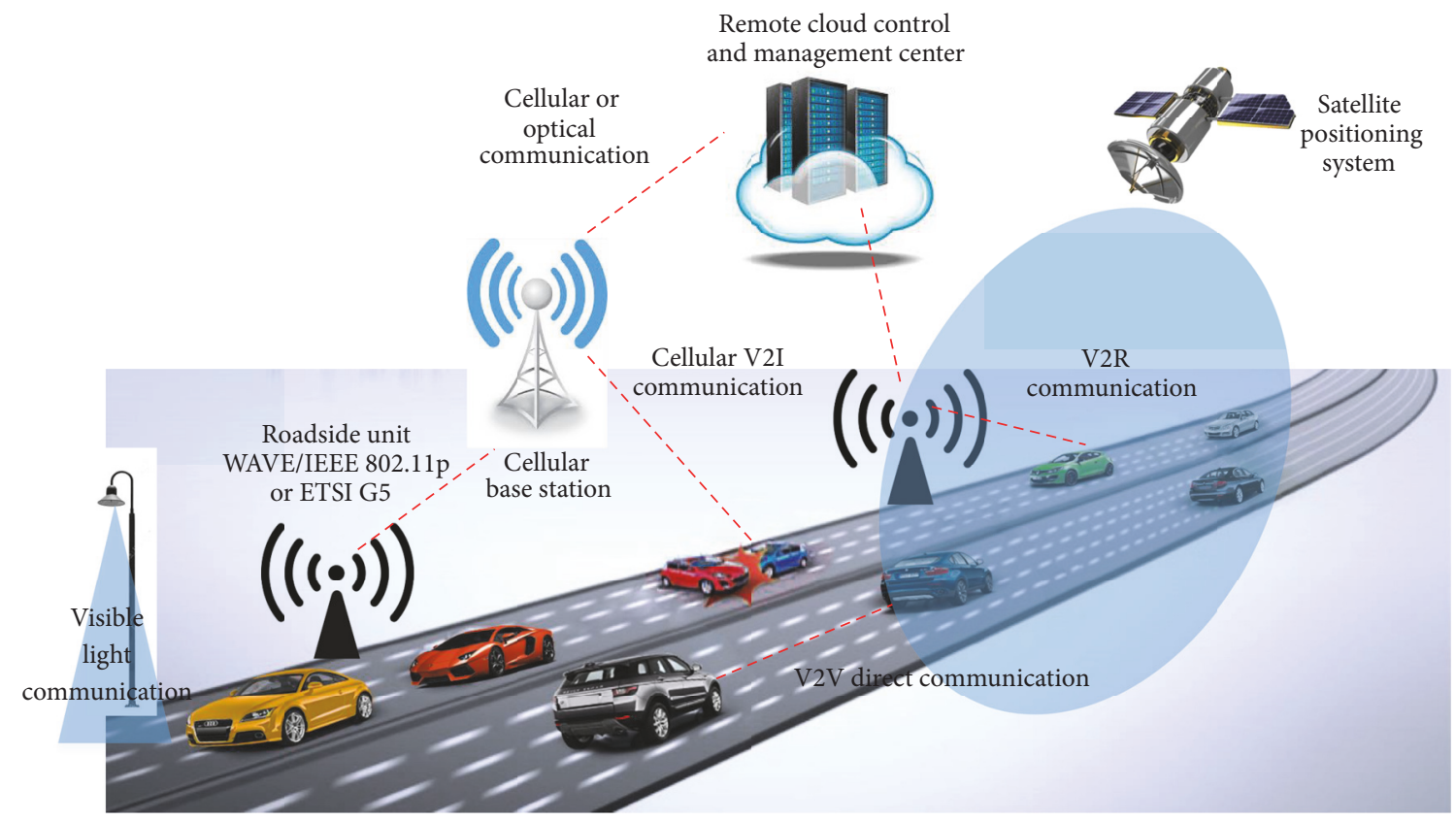

FIGURE 1: General architecture connecting vehicles. The figure shows several strategies for communication: (a) Visible Light Communication; (b) V2R Short Range Communication; (c) WAVE/IEEE 802.11p; (d) Cellular Communication; (e) Vehicle-to-Vehicle Communication; (f) Vehicle-to-RSU Communication; (g) Communication to Remote/Management Centers; (h) Positioning Systems.

(v) Architecture Employing Publicly Available Infrastructure

(vi) Testbeds and Real Deployments

(vii) Virtual Infrastructure

(viii) Hybrid Architectures.

In the remainder of this section, we overview the categories just outlined.

3.1. Analytic Studies Addressing Specific Aspects of the Infrastructure. Abdrabou and Zhuang [86] propose a framework based on the queuing theory that gives a delay bound for relaying messages to RSUs through V2V communication. Authors study the multihop packet delivery delay in a lowdensity vehicular network addressing a disrupted vehicle-toinfrastructure communication scenario where an end-to-end path is unlikely to exist between a vehicle and the nearest RSU.

3.2. Benefits of Incorporating the Infrastructure in Vehicular Networks. Kozat and Tassiulas [10] consider the transport capacity of an ad hoc network with a random flat topology under the presence of an infinite capacity infrastructure network. The main idea is to use the nodes as relays and the infrastructure as pathways to drive the message as fast as possible to the destination node. The results demonstrate a significant improvement achieved using the infrastructure support. Banerjee et al. [12] present a comprehensive comparison between different types of RSUs. Both analytical and simulation results reveal that both the relay and mesh nodes, as opposed to base stations, can be more cost-effective solutions even though a much larger number of such units are required to deliver the same level of performance as offered by the base stations. In addition, authors also suggest that adding a small infrastructure is vastly superior to a large number of mobile nodes with routing capabilities (multihop communications).

Reis et al. [13] study the effects of including RSUs as relay nodes to improve the communication in highway scenarios. Authors model the average time taken to propagate a packet to disconnected nodes when considering both scenarios of connected and disconnected RSUs. The trade-off between the required number of RSUs and the vehicular network performance in sparse scenarios is an important problem that needs careful study. The results show that significant improvements can be achieved with RSUs. For single-gap communications, the transmission delay can be reduced by $15 \%$ to $30 \%$; for traversing multiple gaps, up to $25 \%$ reduction in the endto-end delay with disconnected RSUs is achievable, and with connected RSUs, the decrease in delay can be of several orders of magnitude, depending on the desired area of interest. Authors conclude that sparse scenarios require a strong deployment of RSUs. In scenarios with multiple clusters, the connection between RSUs can greatly reduce the time to transmit information between vehicles. The use of RSUs to solve the disconnected network problem is still an important issue to tackle to be able to provide reliable communications for vehicular applications.

Gerla et al. [11] propose the vehicular grid, a large-scale ad hoc network with ubiquitous presence of the infrastructure. The vehicular grid must be entirely self-supporting for emergency operations (natural disaster, terrorist attack, etc.) and should also exploit the infrastructure during normal operations. The goal of the work is to bring Internet access 
to drivers. Authors argue that the access is possible because every vehicle will be only a few hops away from the infrastructure (Wi-Fi, cellular, etc.). Authors show that routing propagation can be done across the urban environment. Additionally, Gerla and Kleinrock [134] perform a historical comparison of the evolution of the Internet to identify the possible paths to be followed by vehicular communication technologies. Authors argue that the type of assistance requested from the infrastructure will vary according to application. Ideal access points' installations for vehicles are traffic lights, light poles, overpasses, and other public structures. In particular, traffic lights are perfectly positioned to act as traffic routers since they form a traffic grid (i.e., they are placed where traffic is intense) and are equipped with power and directly maintained by local municipalities.

Mershad et al. [14] propose ROAMER (ROAdside Units as MEssage Routers in VANETs) to exploit RSUs to route packets between any source and destination in vehicular networks. The basic motivation behind using RSUs to route packets is that RSUs are stationary. It is much easier to send a packet to a fixed near target than to a remote moving object. ROAMER forwards packets to multiple neighbors to increase the chances of reaching destination without significantly increasing the overall traffic. Authors evaluate the RSU backbone routing performance via the ns- 2 simulation platform and demonstrate the feasibility and efficiency of the scheme in terms of query delay, packet success delivery ratio, and total traffic.

3.3. Cooperative Architectures. Liang and Zhuang [22] propose roadside wireless local area networks (RS-WLANs) as a network infrastructure for data dissemination. More precisely, a two-level cooperative data dissemination approach is proposed. For the network level, the aim is to use available RS-WLANs to provide services to nomadic users. Packet level cooperation uses cooperative caching/transmission to improve the transmission rate: cooperative caching reduces the perception of limited bandwidth whereas cooperative transmission improves the packet transmission rate.

3.4. Light and Smart Architectures. Luan et al. [56] propose an infrastructure composed of roadside buffers, devices with limited buffer storage, and wireless connection to support the vehicular communication with the goal to reduce the costs of network deployment. In addition, Mishra et al. [52] propose the use of stationary info-stations and moving vehicles in a publish-subscribe model. Vehicles may act as publishers, subscribers, or brokers. Every major crossing of city is equipped with stationary info-stations that act as ultimate place holders for publications and subscriptions.

Palazzi et al. [28, 29] investigate an infrastructure for gaming over vehicular networks. They consider the problematic coexistence between TCP and UDP flows in the context of infrastructure-based vehicular networks. They observe that retransmissions of TCP are exacerbated in vehicular networks since the high mobility of vehicles generates continuous variations in the number and type of flows served by the infrastructure along the road. Thus, they propose the use of smart access points along roads, to be able to regulate heterogeneous transmission flows and make them coexist efficiently. Smart access points basically snoop transiting packets of various flows and computes the maximum data rate at which each elastic application will be able to transfer data without incurring in congestion losses. This data rate is computed and also included on the fly in transiting ACKs of TCP flows. They validate their strategy using the ns-2 simulator. Authors use a grid-like road network streaming the video Star Wars IV in high-quality MPEG4 format. Online gaming traffic is inspired by real traces of the popular Counter Strike action game, and it has (i) a server-to-client flow characterized by an interdeparting time of game updates of 200 bytes every $50 \mathrm{~ms}$ and (ii) a client-to-server flow of 42 bytes every $60 \mathrm{~ms}$.

3.5. Architectures Employing Publicly Available Infrastructure. Marfia et al. [57] exploit the use of public Wi-Fi access points to provide vehicular communication. Authors map the public access points available in the city of Portland (US) and vehicles can opportunistically use the infrastructure to communicate with other vehicles in order to avoid long wireless ad hoc paths and to alleviate congestion in the wireless grid. Analytic and simulation models are used to optimize the communications and networking strategies. Authors conclude that the motion model has enormous impact on the results and that the presence of infrastructure largely improves the communication. When the community focuses on the shortness of contact time between vehicles and infrastructure, it becomes clear that scheduling algorithms should consider the data size and deadline or even employ broadcasting to serve a large number of requests. Zhang et al. [58] propose a scheduling scheme for RSUs to provide a balance between serving downloads and upload requests from fast-moving vehicles on highways. The infrastructure acts as routers to Internet access. Although the Internet connection proves to be of great value for drivers, the deployment and maintenance costs of the infrastructure are considered very high. Thus, the authors propose the deployment of cheap RSUs acting as buffers between vehicles.

3.6. Testbeds and Real Deployments. With the aim to create smarter roads by developing an infrastructure that is able to communicate with connected vehicles and with the aim to overcome geographical and standardization boundaries among different countries, Holland, Germany, and Austria developed the so-called Cooperative Intelligent Transport System (C-ITS) Corridor, which represents the first smart highway in Europe [135]. Two applications are implemented: roadworks warning and improved traffic management, both enabled by the cooperation of ETSI ITS-G5 and cellular networks. Roadworks warning aims to improve safety and is enabled by equipping roadworks safety trailers with a positioning/communication system. The roadworks safety trailer continuously transmits its position to a remote control center, where it is evaluated. If available, background information about the roadworks is added and sent back to the roadworks safety trailer. The trailer transmits a warning to approaching vehicles via ETSI ITS-G5. At the same time, the remote 
control center can provide data to a Point of Access, where it is made available for third parties (the Point of Access is, for instance, the Mobility Data Marketplace (MDM) in Germany and the National Data Warehouse (NDW) in Netherlands).

The traffic management service, instead, aims at improving traffic management by highway operators: vehicles send messages to an ETSI ITS-G5 RSU, which then preprocesses the data and forwards it to the traffic control center via cellular network. These messages are standardized Cooperative Awareness Messages (CAM) and Decentralized Environmental Notification by Messages (DENM). CAM are sent continuously. They contain information about the current position of a vehicle, its speed, direction, and dimensions. DENM are sent event-driven when the vehicle detects ice, a traffic jam, or a broken-down vehicle, for example. The C-ITS Corridor represents the first real development given by a close cooperation between road operators and vehicle manufacturers (BMW, Daimler, Ford Deutschland, Adam Opel, and Volkswagen as well as the German Association of the Automotive Industry) [136].

In August 2012, the University of Michigan launched the Connected Vehicle Safety Pilot Model Deployment, the US's largest test of the potential of connected vehicles technologies. The project involved nearly 3,000 private cars, trucks, and buses equipped with OBUs to allow wireless communication with each other and with devices in the roadway infrastructure of northeast Ann Arbor. Communication was enabled by DSRC at $5.9 \mathrm{GHz}$ [137]. The project is expanding to include up to 9,000 equipped vehicles, a back-haul communications network, and back-end data storage. Each OBU accumulates data at the rate of 10 times per second, allowing researchers to test connected vehicle operations for the applications of traffic efficiency, energy efficiency, and environmental benefits. Huge investments on connected vehicles are also done in Japan [138], where great attention on road safety and traffic management has been paid since 1998 [139] to deploy efficient roads and safer vehicles.

$\mathrm{Wu}$ et al. [140] propose a real testbed for evaluating communication between moving vehicles and infrastructure. Authors exploit the communication between vehicles and the infrastructure. Opportunistic forwarding (store-carryforward) appears to be a viable approach for data dissemination using vehicle-to-vehicle communications for applications that can tolerate some data loss and delay. Studies show that $\mathrm{V} 2 \mathrm{~V}$ communication is feasible, although the propagation performance depends on factors such as the density of instrumented vehicles along the end-to-end path. Authors propose the infrastructure to reduce path vulnerability in critical areas or in a subset of vehicles equipped with cellular messaging systems. Field experiments were conducted using a laptop, 802.11b (IEEE 802.11 Wireless Local Area Networks Working Group; http://www.ieee802.org/11/) card with a $2.5 \mathrm{~dB}$ omnidirectional external antenna placed on the roof of the vehicle and a GPS receiver. They measured the wireless communication performance between a fixed roadside station and a moving vehicle. Most of the measurements show more than $500 \mathrm{~m}$ of effective communication range. They also measured communication performance between two vehicles traveling in opposite directions. Most test cases showed more than $200 \mathrm{~m}$ of effective communication range. Average time for effective communication is about $21 \mathrm{~s}$. Authors conclude that vehicular communication is feasible.

Ormont et al. [74] mounted a testbed in the city of Madison, Wisconsin, to monitor Wi-Fi signals over the city. The main communication channel is the $3 \mathrm{G}$ cellular network. Clients were installed in two buses. Each city bus operates on multiple routes on a single day and is, therefore, able to traverse through significant parts of the city. Buses provide Internet access to passengers through $3 \mathrm{G}$ connection. A client is a laptop with a Wi-Fi interface running software that monitors and stores Wi-Fi networks found. Cellular interface provides continuous remote access to each testbed node to experimenters. A client node uses it to periodically upload measurement data to a back-end database system. Authors argue that such testbed can be used to draw coverage maps, analyze performance at specific locations, infer mobility patterns, and study relationships between performance and mobility.

Ruiz et al. [46] study the handover using WiMAX (IEEE 802.16; http://wirelessman.org/) and Wi-Fi applied to vehicular communication. They have mounted a testbed in the Campus of Espinardo, University of Murcia. Campus has a ring road that surrounds a huge enough building area. Any vehicle connected to the wireless network can freely move, using different access points that could be available throughout its path. These access points could belong to different domains and different wireless technologies like Wi-Fi, WiMAX, and Universal Mobile Telecommunication System (UMTS) (http://www.protocols.com/pbook/umts.htm). As a consequence of this, several types of handovers can be differentiated:

(i) Intradomain intratechnology handover

(ii) Intradomain intertechnology handover

(iii) Interdomain intratechnology handover

(iv) Interdomain intertechnology handover.

Authors use the Mobile Internet Protocol for IPv6 (MIPv6) (MIPL Mobile IPv6 Home Page; http://mobile-ipv6 .org/) in order to make the vehicles change from service providers, but keeping the same IP address. They conclude that the deployment of wireless infrastructures must take into account the surrounding environment and the specific circumstances, using the advantages of the different wireless technologies available transparently to the end user.

The following demonstrations, trials, and projects prove how V2X communications based on cellular infrastructure can enable safety applications as well as improved comfort for the driver. 3GPP has in fact recently standardized a set of features that address vehicular communications both for direct (V2V) and for indirect (V2I, V2R) modes. More details on this technology are provided in Section 4.2.

Audi, Vodafone, and Huawei demonstrated vehicular safety applications on the world famous Circuit de BarcelonaCatalunya race track at the Mobile World Congress 2017. The applications were "see through" (connected cars can see a video feed from a vehicle in front of them in situations where it will help them to have visibility of other traffic, upcoming 
entry roads, or other issues to negotiate); a traffic light warning (traffic light is about to change alerting the driver to slow down), pedestrian in the roadway warning; and emergency braking warning (other connected vehicles suddenly braking or changing lanes) [141].

In January 2017, Audi, Ericsson, Qualcomm, Swarco, and Kaiserslautern University announced the formation of Connected Vehicle to Everything of Tomorrow (ConVeX), that is, a consortium to carry out a V2X trial to evaluate range, reliability, and latency of LTE-based V2X communications. Additionally, the trial aimed at highlighting new use cases that help support traffic flow optimization and improve safety. ConVeX plans to use the results of the trial to inform regulators, provide important inputs to ongoing global standardization work, and shape a path for further development and future evolution of Cellular-V2X [142].

Ericsson, Orange, and PSA Group have also planned field trials to test advanced Cellular-V2X applications in France, starting in February 2017. The initial phase of testing focused on two use cases: "see through" between two connected vehicles on a road and "emergency vehicle approaching," aiming at notifying drivers when an emergency vehicle is nearby in real time. These two use cases rely on low latency and high throughput, given that two vehicles need to directly exchange a high-resolution video stream [143].

Vodafone, Bosch, and Huawei are currently working on a trial in the stretch of the A9 Nuremberg and Munich in Germany in the context of a project called Mobilfunk. During the trial, the consortium is demonstrating the viability of direct V2V communications and the ability to exhibit very low latency. In addition, the tests are intended to investigate how Cellular-V2X differs from the IEEE 802.11p [144].

Jaguar Land Rover, Vodafone, and other partners are currently involved in a project called Connected Intelligent Transport Environment (UKCITE) to create an environment for testing connected and autonomous vehicles. It involves equipping over 40 miles of urban roads, dual-carriageways, and motorways with various V2X technologies. The project establishes how this technology can improve journeys; reduce traffic congestion; and provide entertainment and safety services through better connectivity [145].

Audi, Deutsche Telekom, Huawei, and Toyota are conducting trials of Cellular-V2X technology on a section of the "digital A9 motorway testbed" near Ingolstadt, Germany. Audi AG and Toyota Motor Europe research cars and Deutsche Telekom infrastructure have been specially equipped with V2X hardware from Huawei to support the trial scenarios [146].

Continental, DT/T-Systems, Nokia, and Fraunhofer have demonstrated with a trial that vehicles on the motorway can share hazard information using Deutsche Telekom's LTE network. As extremely short transmission times are vital for this purpose, a section of the Deutsche Telekom network was equipped with innovative Mobile Edge Computing technology from Nokia Networks and upgraded with positionlocating technology developed by Fraunhofer ESK. This combination permitted indirect signal transport times between two vehicles of less than 20 milliseconds [147].
3.7. Virtual Infrastructure. Jerbi et al. [50] observe that the need for an infrastructure can decrease the area of vehicular network applications. Therefore, the authors propose a selforganizing mechanism to emulate a geolocalized virtual infrastructure in order to avoid the costs of the deployment. To this purpose, vehicles currently populating the geographic region are used. The geolocalized virtual infrastructure mechanism consists in electing vehicles that will perpetuate information broadcasting within the intersection area. The geolocalized virtual infrastructure is composed of two phases: (i) select vehicles able to reach the broadcast area and (ii) only one among the selected vehicles is elected as the local broadcaster. The elected vehicle performs a local/single hop broadcast once it reaches the broadcast area. Authors conclude that the proposed geolocalized virtual infrastructure can (i) periodically disseminate the data within a given area; (ii) efficiently utilize the limited bandwidth; and (iii) ensure a high delivery ratio.

Luo et al. [51] propose MI-VANET (Mobile Infrastructure-based VANET), a two-tier architecture: buses constitute the mobile backbone for data delivery, while the low tier is composed of ordinary cars and passengers. Cars must register in buses in order to send/receive data. There is a score mechanism to choose the best bus. When the car is leaving the communication range of its registered bus, another bus will be chosen for registration. Authors use VanetMobiSim as a traffic simulator, and they assume that (i) vehicles are uniformly distributed over the road and (ii) buses represent $20 \%$ of the vehicles. Routing algorithm used on the high tier is called Mobile Infrastructure Routing. Each bus knows its location and has a digital street map including bus line information. MIRT is a location based reactive routing protocol that selects the optimal route and forwards the request hop-by-hop. Simulation results show that there is a $40-55 \%$ improvement in delivery ratio while the throughput is even doubled compared to greedy perimeter stateless routing (GPSR) for wireless networks (http://www.cs .cmu.edu/ ./bkarp/gpsr/gpsr.html) in traditional vehicular networks.

Annese et al. [44] study the vehicle-to-infrastructure communication to provide UDP-based multimedia streams. The work considers continuous coverage by the infrastructure within the urban road topology and analyzes the vehicular communication as a mesh network [148]. Mesh networks are typically free-standing robust systems that can be conveniently integrated with the existing infrastructure and offer high bit rate services. Authors do not assume vehicles as end nodes (such as those proposed in [31-33, 73]), but as mesh nodes connecting the wireless medium and acting as routers. They argue that such new point of view is important because it allows the routing protocol to run on the mobile node itself, better adapting to the high-mobility profile of the node. The vehicle becomes a mobile hot spot that can act as a gateway towards the mesh infrastructure.

Because of the high investments required to deploy RSUs in large cities, Tonguz and Viriyasitavat [53] propose an alternative approach to roadside infrastructure by leveraging the use of existing DSRC-equipped vehicles to provide RSU's functionality. The approach employs a self-organizing 
network paradigm and draws its inspiration from social biological colonies such as ants, bees, birds, and fishes. Such approach was formulated for the first time by Tonguz [94]. Vehicles acting as temporary RSUs must make brief stops during which they act as communication bridges for other vehicles in the network. Each vehicle runs the distributed gift-wrapping algorithm proposed by Viriyasitavat et al. [20]. Upon receiving a message, the vehicle determines whether it lies on the boundary of a coverage polygon. As a drawback, vehicles acting as temporary RSUs need to make brief stops (approximately $30 \mathrm{~s}$ ) to reach the maximum number of uninformed vehicles. Authors argue that such increase in travel time is small when compared to increases due to accident-induced congestion. Finally, Sommer et al. [54] propose utilizing parked vehicles as relay nodes to address the disconnected network problem. Extensive simulations and real life experiments show that parked cars can increase cooperative awareness by over $40 \%$.

3.8. Hybrid Architectures. Silva and Meira [55] propose integrating stationary RSUs and mobile RSUs into a single architecture. They argue that traffic presents fluctuations according to the type and time of day, weather conditions, events, road works, and accidents. An architecture composed just of stationary RSUs might not thus be able to properly support the network operation all the time. Similarly, an architecture composed just of mobile RSUs may lack part of the robustness provided by stationary RSUs. Furthermore, the traffic fluctuations are limited by the underlying road network, and road networks do not change as often as traffic does [149]. Therefore, it seems straightforward to assume that a set of RSUs will be stationary, while other RSUs will roam in order to meet the traffic changes.

Since major roads account for a higher transportation capacity, that is, they tend to be very popular routes, they are natural candidates for receiving the stationary RSUs. On the other hand, mobile RSUs may be assigned for handling secondary roads: during rush hours, the major roads get congested, and the drivers use secondary roads as an alternative route for escaping congestions, turning the secondary roads into a candidate for receiving temporary support from mobile-and-virtual RSUs, such as drones launched by the stationary RSUs. When considering the functionality, stationary RSUs act as a main backbone for data dissemination by covering the most important regions of an urban area (i.e., regions known as always presenting relevant traffic), while mobile RSUs provide a temporary support for the dissemination of traffic announcements. The results demonstrate that (i) the hybrid architecture improves the number of distinct vehicles experiencing V2I (vehicle-to-infra) contacts up to $45 \%$ and (ii) the feasibility of incorporating mobile RSUs within public transportation vehicles and drones, since the mobile RSUs must travel at speeds ranging from $5.2 \mathrm{~km} / \mathrm{h}$ up to $11.3 \mathrm{~km} / \mathrm{h}$.

\section{Communication in Infrastructure-Based Vehicular Networks}

To address the requirements foreseen by future vehicular networks, different communication modes and technologies have to be adopted, so that the best radio access technology can be used depending on the applications requirements or on technology availability. Figure 1 represents different communication modes needed in a vehicular environment, such as wide area cellular, V2I, V2V, and V2R [3, 150, 151].

An overview of the key C-ITS and DSRC protocols from a standardization perspective is provided in [152] where the road to $5 \mathrm{G}$ is also sketched.

This section provides a summary of the currently available wireless communication standards for V2V, V2R, and V2I. The following subsections then present efforts in addressing communication in infrastructure-based vehicular networks.

4.1. IEEE WAVE and ETSI ITS-G5. In October 1999, the Federal Communications Commission (FCC) in US allocated $75 \mathrm{MHz}$ of spectrum in the $5.9 \mathrm{GHz}$ range $(5825-5925 \mathrm{MHz})$ for Dedicated Short Range Communications (DSRC). This motivated the IEEE standardization body to specify a family of standards called Wireless Access in Vehicular Environments (WAVE) to deliver a communications framework to enable the services such as road safety applications, traffic management, and infotainment. The first trial version of WAVE standard was released in 2006. The IEEE WAVE standard specifies direct communication among vehicles as well as communication between vehicles and the infrastructure. The latter is enabled by deployment of roadside units (RSUs). The V2V mode addresses the need for safety applications which are latency sensitive and allows cars to send to each other periodic updates of their status (i.e., speed and position). Vehicles can communicate directly using the WAVE Short Message Protocols (WSMP). Each vehicle is then connected to the infrastructure through the V2I mode in order to exchange data and control information with the cloud. WAVE enables secure communication and physical access for low latency links, with speeds of up to $27 \mathrm{Mbps}$ across a range of approximately $1000 \mathrm{~m}$. The following standards are part of the IEEE WAVE family:

(i) IEEE 1609 series for architecture, security services for applications and management messages, networking service, multichannel operation, communication manager, over-the-air electronic payment data exchange protocol, and identifier allocations;

(ii) IEEE 1906.4 for MAC layer functions;

(iii) IEEE 802.11p for MAC sublayer management and physical layer.

In the context of DSRC, several research works have been carried out. Campolo et al. [153] present an analytical framework that models the service advertisement and access mechanisms in multichannel vehicular networks. The model accounts for dual-radio devices and computes the mean service discovery time and the service channel utilization. Bazzi et al. [150] demonstrate the impact of number and position of RSUs on the delivery rate of IEEE 802.11p also varying the routing algorithm.

In 2008, the European commission allocated $30 \mathrm{MHz}$ within the $5.9 \mathrm{GHz}$ band for C-ITS wireless communications. 
Following this allocation, the European Telecommunications Standard Institute (ETSI) developed a set of standards for C-ITS, whose objectives are very similar to the ones that motivated the development of WAVE in the US and whose access layer uses a specific set of options of the IEEE 802.11p specifications. IEEE 802.11p in US and ITS-G5 Release 1 in Europe have been considered to date as the de facto standard technologies for vehicular communications at $5.9 \mathrm{GHz}$, but things are now changing since $3 \mathrm{GPP}$ has also standardized a set of communication features for vehicular scenarios, which we refer to as Cellular-V2X, as outlined in Section 4.2.

4.2. 3GPP Cellular-V2X. Cellular systems are nowadays widely recognized as drivers of innovation in a wide range of technical fields, and they today represent the most adopted solution to collect data from vehicles and retransmit them to the network through on-board units. This avoids having to build new set-ups or expensive installations at the roadside. Issues like authorization, authentication, and resource allocation are currently always handled by the wide area network.

Moreover, today 3GPP is playing a key role in specifying new features for supporting a wide range of vehicular modes of communications (V2V, V2I, and V2R). We refer to this set of features as Cellular-V2X technologies. Within the Technical Specification Group (TSG) Services and Architecture (SA), a basic set of requirements to support early CellularV2X applications has been specified in [154] following the studies reported in [16]. These requirements are sufficient for vehicles to directly and periodically exchange their own status information such as position, speed, and heading with neighboring vehicles, pedestrians, and road infrastructure nodes and also address the need to disseminate event-driven warning messages. These are the IEEE WAVE and ETSI CITS main focus safety use cases. To address these use cases, the TSG Radio Access Network (RAN) specified V2V communications within Release 14, which builds on the device-todevice (D2D) communications features specified in Release 12 (where the focus was mainly on public safety type of use cases). Within this framework, a new communication interface called Sidelink (or PC5 interface) was specified in Release 12 [155] as a direct link between devices. Improvements to this interface have been added to [155] within Release 14 to address the V2V use cases in the ITS $5.9 \mathrm{GHz}$ band and more specifically

(i) to handle higher Doppler associated with relative speeds of up to $500 \mathrm{Kmph}$ at $5.9 \mathrm{GHz}$;

(ii) to insert arrangements for scheduling assignment and data resources;

(iii) to introduce a sensing with semi-persistent transmission based mechanism for distributed scheduling.

Two high-level deployment configurations are currently defined: distributed scheduling and eNB scheduling. Both configurations use a dedicated carrier for the V2V link, which is the target ITS $5.9 \mathrm{GHz}$ band, and in both cases GNSS is used for time synchronization. This initial work was completed in September 2016 (see [156], which was used to inform 3GPP and external stakeholders that the specification work for V2V using Sidelink is complete). TSG-RAN then worked on enhancing these specifications by adding support for congestion control; coexistence with other ITS technologies that might be using the $5.9 \mathrm{GHz}$ band; and the V2I interface (work item described in [157]). This work was completed within Release 14, which was frozen in February 2017. This can be considered as the first release of CellularV2X technologies.

In [158], TSG-SA has then defined new service requirements to further enhance 3GPP support for Cellular-V2X in the following areas:

(i) nonsafety V2X services (e.g., connected vehicle, mobile high data rate entertainment, mobile hot spot, office, home, and dynamic digital map update);

(ii) safety-related V2X services (e.g., autonomous driving, car platooning, and priority handling between safety-related V2X services and other services);

(iii) support for V2X services in multiple 3GPP radio access technologies (e.g., LTE and 5G) and networks environments including aspects such as interoperability with non-3GPP V2X technologies (e.g., ITSG5, IEEE 802.11p, and ITS-Connect).

In order to address the requirements envisioned by the new use cases, vehicles require new levels of connectivity and intelligence. All the services in the three outlined areas will be addressed by subsequent 3GPP releases, and the CellularV2X features will continue to seamlessly evolve release after release by addressing new and more stringent requirements on network capacity, coverage, reliability, and latency [159$162]$.

$5 \mathrm{G}$ is currently in the process of being specified by $3 \mathrm{GPP}$ in Release 15 . It is a phased approach, and the first $5 \mathrm{G}$ release will be finished by the end of 2017, with focus on enhanced mobile broadband. Subsequent releases will focus on different use cases that require lower latency and higher reliability. The $5 \mathrm{G}$ new air interface, which is called New Radio (NR) in 3GPP context, will ensure increased performance in terms of throughput, latency, reliability, connectivity, and mobility. The architecture of a $5 \mathrm{G}$ system aims to support the convergence of different applications onto a common network, by flexible usage and configuration of network functions. 5G will help to reach a better coverage through the integration of various access technologies and is envisioned to support higher mobility, for example, $500 \mathrm{~km} / \mathrm{h}$. $5 \mathrm{G}$ is also envisioned to improve network reliability, with a 10-5-packet loss rate for safety-critical services.

Table 1 represents a set of vehicular applications addressed by ETSI C-ITS Release 1 and 3GPP Release 14. As mentioned above, the main focus of these two standards is safety and traffic management. ETSI also specifies requirements for infotainment, which, in the context of 3GPP, are addressed by earlier releases. The table highlights requirements for type of connectivity, beacon periodicity (BP) for periodic exchange of status information, and end-to-end latency. (All the applications are enabled by beacons exchange among vehicles. 
TABLE 1: Applications and requirements for ETSI and 3GPP.

\begin{tabular}{|c|c|c|c|c|}
\hline Application & $\mathrm{V} 2 \mathrm{X}$ & Message type & $\begin{array}{c}\text { Beacon } \\
\text { periodicity } \\
{[\mathrm{Hz}]}\end{array}$ & $\begin{array}{l}\text { End-to-end } \\
\text { latency [ms] }\end{array}$ \\
\hline \multicolumn{5}{|c|}{ ETSI safety } \\
\hline Emergency electronic brake lights & $\mathrm{V} 2 \mathrm{X}$ & Periodic & 10 & 100 \\
\hline $\begin{array}{l}\text { Safety function out of normal condition } \\
\text { warning }\end{array}$ & $\mathrm{V} 2 \mathrm{X}$ & Periodic & 1 & 100 \\
\hline Emergency vehicle warning & $\mathrm{V} 2 \mathrm{X}$ & Periodic & 10 & 100 \\
\hline Slow vehicle & $\mathrm{V} 2 \mathrm{X}$ & Periodic & 2 & 100 \\
\hline Motorcycle warning & $\mathrm{V} 2 \mathrm{X}$ & Periodic & 2 & 100 \\
\hline Vulnerable road user warning & $\mathrm{V} 2 \mathrm{X}$ & Periodic & 1 & 100 \\
\hline Wrong way driving warning & $\mathrm{V} 2 \mathrm{X}$ & Event-driven & 10 & 100 \\
\hline Stationary vehicle warning & $\mathrm{V} 2 \mathrm{X}$ & Event-driven & 10 & 100 \\
\hline Traffic condition warning & $\mathrm{V} 2 \mathrm{X}$ & Event-driven & 10 & N/A \\
\hline Signal violation warning & $\mathrm{V} 2 \mathrm{X}$ & Event-driven & 10 & 100 \\
\hline Roadwork warning & $\mathrm{I} 2 \mathrm{~V}$ & Periodic & 2 & 100 \\
\hline Decentralized floating car data & $\mathrm{V} 2 \mathrm{X}$ & Event-driven & $1-10$ & $\mathrm{~N} / \mathrm{A}$ \\
\hline Precrash sensing warning & $\mathrm{V} 2 \mathrm{X}$ & Event-driven & 10 & 50 \\
\hline Hazardous location notification & $\mathrm{V} 2 \mathrm{X}$ & Event-driven & N/A & \\
\hline \multicolumn{5}{|c|}{ ETSI traffic management } \\
\hline Regulatory speed limit & $\mathrm{I} 2 \mathrm{~V}$ & Event-driven & $1-10$ & 500 \\
\hline Traffic light optimal speed advisory & $\mathrm{I} 2 \mathrm{~V}$ & Periodic & 2 & 100 \\
\hline $\begin{array}{l}\text { Traffic information and recommended } \\
\text { itinerary }\end{array}$ & $\mathrm{I} 2 \mathrm{~V}$ & Periodic & $1-10$ & 500 \\
\hline Enhanced route guidance and navigation & $\mathrm{I} 2 \mathrm{~V}$ & Periodic/event-driven & 1 & 500 \\
\hline Intersection management & $\mathrm{I} 2 \mathrm{~V}$ & Periodic & 1 & 500 \\
\hline Cooperative flexible lane change & $\mathrm{I} 2 \mathrm{~V} / \mathrm{V} 2 \mathrm{~V}$ & Periodic/event-driven & 1 & 500 \\
\hline Limited access warning & $\mathrm{I} 2 \mathrm{~V} / \mathrm{V} 2 \mathrm{~V}$ & Periodic/event-driven & $1-10$ & 500 \\
\hline Electronic toll collection & $\mathrm{I} 2 \mathrm{~V} / \mathrm{V} 2 \mathrm{I}$ & Periodic/event-driven & 1 & 200 \\
\hline Cooperative adaptive cruise control & $\mathrm{V} 2 \mathrm{X}$ & Periodic & 2 & 100 \\
\hline Highway platooning & $\mathrm{V} 2 \mathrm{X}$ & Periodic & 2 & 100 \\
\hline \multicolumn{5}{|c|}{ ETSI infotainment } \\
\hline $\begin{array}{l}\text { Point of interest notification/automatic } \\
\text { access }\end{array}$ & I2V/V2I & Periodic/event-driven & 1 & 500 \\
\hline $\begin{array}{l}\text { Local electronic commerce/instant } \\
\text { messaging }\end{array}$ & $\mathrm{I} 2 \mathrm{~V} / \mathrm{V} 2 \mathrm{I}$ & Periodic/event-driven & 1 & 500 \\
\hline Car rental/sharing assignment/reporting & $\mathrm{I} 2 \mathrm{~V} / \mathrm{V} 2 \mathrm{I}$ & Periodic/event-driven & 1 & 500 \\
\hline $\begin{array}{l}\text { Media downloading/map download and } \\
\text { update }\end{array}$ & I2V/V2I & Periodic/event-driven & 1 & 500 \\
\hline Ecological/economical drive & $\mathrm{I} 2 \mathrm{~V} / \mathrm{V} 2 \mathrm{I}$ & Periodic/event-driven & 1 & 500 \\
\hline $\begin{array}{l}\text { Personal data synchronization/vehicle } \\
\text { relation management }\end{array}$ & $\mathrm{I} 2 \mathrm{~V} / \mathrm{V} 2 \mathrm{I}$ & Periodic/event-driven & 1 & 500 \\
\hline SOS service/stolen vehicle alert & $\mathrm{I} 2 \mathrm{~V} / \mathrm{V} 2 \mathrm{I}$ & Periodic/event-driven & 1 & 500 \\
\hline $\begin{array}{l}\text { Remote diagnosis and just in time repair } \\
\text { notification }\end{array}$ & $\mathrm{I} 2 \mathrm{~V} / \mathrm{V} 2 \mathrm{I}$ & Periodic/event-driven & 1 & 500 \\
\hline $\begin{array}{l}\text { Vehicle data collection for product life } \\
\text { cycle management }\end{array}$ & I2V/V2I & Periodic/event-driven & 1 & 500 \\
\hline Insurance and financial services & $\mathrm{I} 2 \mathrm{~V} / \mathrm{V} 2 \mathrm{I}$ & Periodic/event-driven & 1 & 500 \\
\hline $\begin{array}{l}\text { Fleet management/loading zone } \\
\text { management }\end{array}$ & I2V/V2I & Periodic/event-driven & 1 & 500 \\
\hline $\begin{array}{l}\text { Vehicle software/data provisioning and } \\
\text { update }\end{array}$ & I2V/V2I & Periodic/event-driven & 1 & 500 \\
\hline Vehicle and RSU data calibration & $\mathrm{I} 2 \mathrm{~V} / \mathrm{V} 2 \mathrm{I}$ & Periodic/event-driven & 1 & 500 \\
\hline
\end{tabular}


TABLE 1: Continued.

\begin{tabular}{|c|c|c|c|c|}
\hline Application & V2X & Message type & $\begin{array}{c}\text { Beacon } \\
\text { periodicity } \\
{[\mathrm{Hz}]}\end{array}$ & $\begin{array}{l}\text { End-to-end } \\
\text { latency [ms] }\end{array}$ \\
\hline \multicolumn{5}{|c|}{ 3GPP safety } \\
\hline V2I emergency stop use case & V2I & Periodic & 10 & 100 \\
\hline Queue warning & V2X & Periodic & N/A & 100 \\
\hline Road safety services & V2I & Periodic/event-driven & 10 & 100 \\
\hline Wrong way driving warning & $\begin{array}{l}\text { Periodic/event- } \\
\text { driven }\end{array}$ & N/A & N/A & \\
\hline Precrash sensing warning & Event-driven & N/A & 20 & \\
\hline $\mathrm{V} 2 \mathrm{X}$ in areas outside network coverage & Event-driven & N/A & N/A & \\
\hline $\mathrm{V} 2 \mathrm{X}$ road safety service via infrastructure & Event-driven & N/A & N/A & \\
\hline Curve speed warning & V2I & Periodic & 1 & 1000 \\
\hline $\begin{array}{l}\text { Warning to pedestrian against pedestrian } \\
\text { collision }\end{array}$ & $\mathrm{V} 2 \mathrm{X}$ & Periodic & N/A & N/A \\
\hline \multicolumn{5}{|c|}{ 3GPP traffic management } \\
\hline Automated parking system & V2X & Event-driven & N/A & 100 \\
\hline
\end{tabular}

Beacons packets are typically short and contain basic information such as the vehicle identification, position, speed, and acceleration. Hence, by exchanging beacons, vehicles become aware of the environment: more frequent beacons mean more consciousness of each own neighbor but higher channel load and risk of collisions.) Please note that the mode infrastructure to vehicle (I2V) has been used to identify those use cases that require updates being broadcast by the network to the vehicles.

4.3. Visible Light Communications: The IEEE 802.15.7 Standard. The great development and deployment of LEDs lights have increased the interest in Visible Light Communications (VLC) technology and to a recent standardization activity, namely, the IEEE 802.15.7 standardization group, which explicitly considers vehicles and illuminated roadside devices (such as traffic lights or street lights) among the addressed applications [163]. The IEEE 802.15.7 specification defines three different PHY levels, with a number of possible modulations and coding schemes, that support data rate ranging from $11.67 \mathrm{~kb} / \mathrm{s}$ to $96 \mathrm{Mb} / \mathrm{s}$. Since the specifications suggest only using PHY I in outdoor applications, the maximum data rate for vehicular communications is however presently limited to a maximum of $266.6 \mathrm{~kb} / \mathrm{s}$. At the MAC layer four options are foreseen by IEEE 802.15.7: either beacon enabled slotted random access or nonbeacon enabled unslotted random access, both with or without carrier sensing multiple access with collision avoidance (CSMA/CA). In VVLNs, nonbeacon enabled unsolved random access without CSMA/CA seems the preferable solution in most cases. At the same time, carrier sensing allows higher throughput and the increasing complexity required for its implementation does not appear as a problem in the vehicular scenario. Table 2 summarizes the main characteristics of the wireless access technologies for vehicular networks.

The following subsections present efforts addressing communication in infrastructure-based vehicular networks.
We identify the following major categories of works composing the communication efforts:

(i) Analytic Studies Addressing Specific Properties of Vehicle-to-Infrastructure Communication

(ii) Strategies for Data Dissemination in InfrastructureBased Vehicular Networks

(iii) Protocols for Infrastructure-Based Vehicular Networks

(iv) Routing in Infrastructure-Based Vehicular Networks

(v) Managing Infrastructure-Based Vehicular Networks

(vi) Privacy and Security in Infrastructure-Based Vehicular Networks

4.4. Analytic Studies Addressing Specific Properties of Vehicleto-Infrastructure Communication. Ng et al. [84] study the access probability considering an infrastructure wherein a number of base stations are uniformly deployed along a long road, while other vehicles or cars are distributed on the road according to a Poisson distribution. The authors formulate a mathematical model that relates the density of vehicles, coverage range, and distance between adjacent base stations to infer the access probability. No measures of delay are given, and opposite-lane message relaying is not considered.

$\mathrm{Ng}$ and Mao [83] analyze the probability of $k$-hops connectivity in infrastructured wireless networks, while Malandrino et al. [23] address content downloading in vehicular networks leveraging both infrastructure-to-vehicle and vehicle-to-vehicle communication. Authors formulate a maxflow problem that accounts for practical aspects, including channel contention and the data transfer paradigm. The goal of the paper is to answer the following question: "what is the maximum downloading performance theoretically achievable through DSRC-based I2V/V2V communication in 
TABLE 2: Main characteristics of wireless access for vehicular networks.

\begin{tabular}{|c|c|c|c|c|}
\hline & $\begin{array}{c}\text { WAVE IEEE } \\
802.11 \mathrm{p} \\
\end{array}$ & 3GPP LTE-A & IEEE 802.15.7 (VLC) & $5 \mathrm{G}$ \\
\hline Frequency & $5.9 \mathrm{GHz}$ & $400 \mathrm{MHz}-3.5 \mathrm{GHz}$ & $380-800 \mathrm{THz}$ & $\begin{array}{l}\text { New bands } \\
\text { between } \\
700 \mathrm{MHz} \text { and } \\
100 \mathrm{GHz}\end{array}$ \\
\hline \multirow{2}{*}{ Data rate } & \multirow{2}{*}{$27 \mathrm{Mb} / \mathrm{s}$} & $3 \mathrm{~Gb} / \mathrm{s}$ (downlink) & \multirow{2}{*}{$11.67 \mathrm{~kb} / \mathrm{s}-96 \mathrm{Mb} / \mathrm{s}$} & \multirow{2}{*}{$\begin{array}{l}\text { Up to } 1000 \\
\text { times greater }\end{array}$} \\
\hline & & $1.5 \mathrm{~Gb} / \mathrm{s}$ (uplink) & & \\
\hline $\begin{array}{l}\text { Communication } \\
\text { range }\end{array}$ & $<1000 \mathrm{~m}$ & Ubiquitous & $<100 \mathrm{~m}$ & Ubiquitous \\
\hline Latency & $<100 \mathrm{~ms}$ & $<100 \mathrm{~ms}$ & $<50 \mathrm{~ms}$ & $<10 \mathrm{~ms}$ \\
\hline Mobility speed & $<300 \mathrm{~km} / \mathrm{h}$ & $<300 \mathrm{~km} / \mathrm{h}$ & N/A & $<500 \mathrm{~km} / \mathrm{h}$ \\
\hline $\begin{array}{l}\text { V2I } \\
\text { communication }\end{array}$ & $\begin{array}{c}\text { Supported after } \\
\text { deployment of } \\
\text { RSUs }\end{array}$ & Supported & $\begin{array}{l}\text { May use the available roads } \\
\text { lights }\end{array}$ & $\begin{array}{l}\text { Networks } \\
\text { cooperation }\end{array}$ \\
\hline $\begin{array}{l}\mathrm{V} 2 \mathrm{~V} \\
\text { communication }\end{array}$ & Supported & $\begin{array}{c}\text { Supported from Release } 14 \\
\text { in } 5.9 \mathrm{GHz}\end{array}$ & Supported & $\begin{array}{c}\text { Networks } \\
\text { cooperation }\end{array}$ \\
\hline Deployment & $\begin{array}{l}\text { Requiring RSUs } \\
\text { set-up }\end{array}$ & $\begin{array}{c}\text { May use the available } \\
\text { eNodes B }\end{array}$ & May use the available LEDs & $\begin{array}{c}\text { Upper layers } \\
\text { cooperation and } \\
\text { management }\end{array}$ \\
\hline
\end{tabular}

a given mobility scenario?" Authors conclude that a densitybased RSUs deployment yields performance close to optimum and that multihop traffic delivery is beneficial, although the gain is negligible beyond two hops away from the RSU.

Zhang et al. [49] propose an analytical model to predict both the uplink and downlink connectivity probabilities. The uplink connectivity probability is defined as the probability that messages from vehicles can be received by the infrastructure through multihop paths. The downlink connectivity probability is defined as the probability that messages can be broadcast from RSUs to all vehicles through multihop paths. Abdrabou and Zhuang [82] propose an analytic framework that helps to approximately estimate the minimum number of RSUs required to cover a road segment with a probabilistic vehicle-to-infrastructure delay guarantee, given that intermittent multihop connectivity exists between vehicles and RSUs, and vehicles are sending bursty traffic. In [42], the authors present a study of the relation between packet delivery delay and RSU density for vehicular-toinfrastructure communication in sparse vehicular networks where vehicles moving in one direction send their packets to vehicles traveling in the opposite direction in order to deliver the packets to the nearest RSU.

Lochert et al. [25] discuss the initial moments of a vehicular network and they demonstrate that during the rollout phase some kind of support is needed. Otherwise, many envisioned applications are unlikely to work until a large fraction of vehicles participate in the network. Authors use stationary support units to improve the refreshing rate of the information dissemination in city scenarios.

4.5. Strategies for Data Dissemination in Infrastructure-Based Vehicular Networks. Data dissemination is at the basis of most services enabled by vehicular networks and its importance is demonstrated by the large number of papers in the literature addressing this issue. In [164], for example, twentythree different kinds of dissemination schemes have been reviewed and a comparative analysis is provided highlighting the benefits and drawbacks associated with each scheme.

Liu and Lee [41] propose push-based broadcast data dissemination in heavy traffic: messages are periodically broadcast to passing vehicles. In light traffic scenarios, vehicles query on-demand traffic information. Authors derive a mathematical model that shows the effectiveness of their solution and they conclude that data dissemination in vehicular networks should be adaptable to dynamic traffic environments: dynamic channel and data allocation is a critical, but an effective mechanism in providing hybrid scheduling between push-based and on-demand services.

Bruno and Nurchis [26] assume vehicles equipped with cameras and the problem is how to deliver the images to remote data collectors. Authors propose a data collection algorithm capable of eliminating the redundancy of data transmitted by moving vehicles. In a real situation, several vehicles may report the same event. Thus, data redundancy mitigation is necessary to improve the network efficiency. The model is based on the Maximum Coverage Problem [165] followed by submodular optimization.

Data scheduling is also addressed in [27], where the authors propose a downlink scheduler to deliver high-quality video-on-demand services over infrastructure-based vehicular networks. The scheduler is deployed at RSUs to coordinate the transmission of packets according to (i) importance of packet to video quality; (ii) playback deadline; and (iii) realtime information of vehicles.

Zhang et al. [166] also devise a scheduling algorithm to coordinate the distribution of data files in vehicular networks. A collection of data files are stored at distributed locations and delivered to passing vehicles. According to the popularity of files, the proposed algorithm schedules the location of 
files through the selective upload and download of RSUs to maximize the delivery ratio of files to vehicles.

4.6. Protocols for Infrastructure-Based Vehicular Networks. Hadaller et al. [61] propose MV-MAX (Multi-Vehicular Maximum), a protocol to increase the global data transfer. Authors observe that when a RSU is shared by more than one vehicle, the vehicle with the lowest transmission rate reduces the effective transmission rate of all other vehicles. Observing that every vehicle eventually receives good performance when it is near the RSU, authors propose a medium access protocol that opportunistically grants access to vehicles with maximum transmission rate. The overall system throughput is improved by a factor of four.

Korkmaz et al. [63] propose a cross-layer multihop data delivery protocol with fairness guarantees where vehicles do not communicate with RSUs individually, but through one leader. The goal is to reduce the network traffic and to use bandwidth more efficiently. The leader will collect all information from other nodes and share it with RSUs.

Korkmaz et al. [40] propose a new protocol that employs fixed gateways along the road which perform periodic admission control and scheduling decisions for the packet traffic in their service area. The most important contribution of the protocol is providing delay bounded throughput guarantees for soft real-time traffic, which is an important challenge especially for a mobile multihop network. After the demands of the soft real-time traffic are met, the protocol supports the best-effort traffic using remaining bandwidth.

Ramani and Savage [31] propose SyncScan to continuously track nearby base stations by synchronizing short listening periods at the client with periodic transmissions from each base station. Brik et al. [32] propose MultiScan so that nodes rely on using their (potentially idle) second wireless interface to opportunistically scan and preassociate with alternate access points and eventually seamlessly hand-off ongoing connections.

4.7. Routing in Infrastructure-Based Vehicular Networks. A careful review of the main routing algorithms revealed that most proposals are inspired by either greedy forwarding (GF) or distance vector (DV): the former exploits the actual position of each node to find the route with the minimum source-destination distance, while the second finds the route with the minimum number of hops between the source and the destination. Since GF always tries to get closer to the destination, it appears more suitable for delay tolerant and not fully connected networks. On the other hand, DV also supports real-time communications.

Borsetti and Gozalvez [43] propose an infrastructureassisted routing approach designed to improve the end-toend performance, range, and operation of multihop vehicular communications by exploiting the reliable interconnection of infrastructure units. The infrastructure is wired-connected and uses the position of each vehicle to route the data. Authors use SUMO traces and a grid streets layout. They conclude that to obtain the maximum benefits from the proposed infrastructure-assisted routing approach, optimal infrastructure deployment strategies must be further investigated.

In [123], the well-known Ad hoc On-demand Distance Vector (AODV) routing protocol is modified by replacing the flooding mechanism, used in its route discovery process, with the probabilistic forwarding technique given by Irresponsible Forwarding (IF) [167]. The performance of the new routing protocol, denoted as irresponsible AODV (iAODV), is analyzed in three characteristic scenarios (pedestrian, pedestrian-vehicular, and vehicular). The obtained results show that the iAODV protocol can outperform the AODV protocol by significantly reducing the overhead traffic during the route discovery phase. In particular, iAODV takes advantage of, rather than combating, high node spatial density and/or data traffic load.

A promising solution to the information dissemination in urban environments is the intersection-based geographic routing protocol. In [168], four issues (i.e., intermittent connectivity, traffic light at intersections, and three-dimensional and traffic accident city scenarios) which strongly affect the performance of geographic routing protocols are addressed and their impact on the performance of routing protocols is demonstrated, suggesting important guidelines for network designers.

4.8. Managing Infrastructure-Based Vehicular Networks. The ultimate goal of a vehicular network is to serve as a communication layer for vehicular applications. There are several envisioned vehicular applications: monitoring roads conditions [66], vehicles' performance [169], driver's behavior $[170]$, routes optimization $[171,172]$, smart traffic lights [149, 173], traffic monitoring [68], collaborative driving [69], and accident detection [174, 175] offering a large spectrum of traffic information solutions demanding minimal (and possibly distinct) QoS guarantees that must couple the vehicular network.

Traditional strategies for measuring the network performance are based on the network latency and bandwidth. However, when we consider mobile nodes, the latency and bandwidth become location-dependent, since both measurements fluctuate according to the nodes' locations. Similarly, typical metrics adopted in managing cellular networks do not seem to fully qualify for vehicular networks. However, we are not supposed to experience large-scale vehicular networks until we learn how to manage such networks. Metrics are the basis for defining Service Level Agreements that will guide the operation of vehicular networks. They will also indicate when the network demands upgrade and where the upgrade is supposed to take place.

We have captured two works addressing the QoS for lower layers: Luan et al. [36] focus on the MAC layer for V2I communications where multiple fast-moving vehicles with different on-top applications and QoS requirements compete for the transmissions to the roadside infrastructure, while Harigovindan et al. [21] develop a mechanism for fair channel allocation.

From a complementary perspective, there are also works proposing strategies based on higher layers. Silva and Meira [37] propose the Delta Network as a strategy to reflect 
the connectivity experienced by vehicles. Delta is based on two measurements: (i) connectivity duration and (ii) percentage of vehicles presenting such connectivity duration. For instance, if a given vehicular network provides $20 \%$ of all vehicles connected during $30 \%$ of the trip duration, such network is considered a $\Delta_{0.2}^{0.3}$ Network. The authors argue that the Delta Network can be used to (i) support the design of new vehicular networks; (ii) compare the performance of distinct vehicular networks; and (iii) evaluate the adherence between vehicular applications and the network.

When Delta measures the time duration that vehicles are connected (only) to RSUs, the metric turns into a strategy for evaluating the quality of service provided by a given deployment of RSUs. However, in case we use Delta to measure the time duration that vehicles are connected between themselves, the metric becomes a strategy to measure the quality of service provided by the ad hoc V2V communication. We can also use Delta as a deployment strategy by formulating an optimization problem stating "how many RSUs are required (and where must they be deployed) so that we can achieve a vehicular network allowing that $\rho_{2}$ percent of the vehicles are connected to RSUs during $\rho_{1}$ percent of the trip duration?" In other words, we intend to find out how many RSUs are required to achieve a $\Delta_{\rho_{2}}^{\rho_{1}}$ Network.

The time duration that vehicles are connected is an important measurement. However, we must also characterize the interconnection gap (the time duration that vehicles are not connected). This is the goal of the Gamma Network proposed in $[38,39]$. Gamma is a strategy for planning the roadside infrastructure in order to achieve predefined levels of service in terms of the interconnection gap and the share of vehicles experiencing such interconnection gap. A given layout of RSUs is considered $\Gamma_{\mathrm{D}}\left(\begin{array}{c}\tau \\ \rho\end{array}\right)$ whenever $\rho$ percent of all vehicles are guaranteed to meet RSUs in intervals less than (or equal to) $\tau$ seconds over the entire trip.

4.9. Privacy and Security in Infrastructure-Based Vehicular Networks. Extensive researches are being carried on to provide security and privacy in vehicular networks so that the true identity of the drivers and sensitive information are not exposed. Hence, the security and privacy issues must be handled carefully so that the adversaries cannot misuse them [176].

Plobl and Federrath [47] propose a set of security requirements for the infrastructure of vehicular networks in terms of integrity, confidentiality, and availability. In order to protect the integrity, the security infrastructure has to provide mechanisms that prevent and detect the modification of messages. Furthermore, the authenticity and integrity of the message must be provable instantly without further information. Proof of integrity and origin of data are recommended to prevent misuse of the network combined with correct time and position information in all messages to protect against replay and position spoofing attacks.

In terms of confidentiality, the security infrastructure also has to provide mechanisms that support different levels of confidentiality, and all messages should be protected against eavesdropping. In terms of availability, the network must provide real-time processing of messages, possibly using data compression techniques to reduce network bandwidth consumption combined with actions to complicate denialof-service attacks. Authors propose that, after a once-only initialization, the system employs asymmetric cryptography within a public key infrastructure for messages influencing road safety. All other messages are protected by a system employing symmetric cryptography.

Gómez Mármol and Martínez Pérez [45] propose TRIP (Trust and Reputation Infrastructure-based Proposal), a model used to decide whether to accept a traffic warning coming from another vehicle or not by assessing the trustworthiness of the issuer of such message. Authors extend the requirements of the vehicular infrastructure by proposing that the security infrastructure should also be able to make fast decisions to deal with the constantly changing topology and fast switching of neighbors; otherwise, the communication becomes very inefficient. Network should also be resilient to security and privacy threats such as malicious nodes trying to drive the reputation of a reliable node down. Finally, the security must also be independent of mobility patterns in order to accurately perform under every possible traffic scenario.

As another example, the actors of the European CITS Corridor [135], which involves three countries in the experiment, faced the issues of confidentiality and availability from the early stages of the trial: for the roadworks warning application specific data protection laws were not necessary, whereas for cooperative traffic management with the inclusion of vehicle data, privacy protection has been applied to grant anonymity still providing some security to verify the authenticity of messages.

Oliveira et al. [48] propose a Social Network for Vehicular Certification (SNVC) for the exchange of cryptographic material in daily relationships. The SNVC establishes trust degrees among users in the social network and a reputation mechanism allows tracking the reputation of users. The reputation mechanism can identify users that collaborate in the generation of reliable information at the cyber-physical Mobile Opportunistic Network.

\section{Deployment of Infrastructure for Vehicular Networks}

The deployment of infrastructure is one of the most critical decisions when designing vehicular networks. Deployment is the task of defining the exact location of RSUs within the road network. A misleading deployment incurs in waste of valuable resources and degradation of the network performance. So far, the deployment of infrastructure for vehicular networks is an open problem since it depends on the intrinsic mobility of the network nodes and also on the applications running on top of the vehicular network. Although the deployment of RSUs may resemble the deployment of base stations in cellular networks, there are several differences. While cellular networks use massive deployments, vehicular networks do not necessarily demand ubiquitous coverage. Instead, vehicles may engage in "infofueling" as they opportunistically drive through RSUs [177]. 
There are also important differences in terms of the mobility of nodes in vehicular networks and cellular networks. In cellular networks, the network designer starts a deployment plan by understanding how the population is distributed over the city. On the other hand, the vehicular networks designer must start by understanding the urban mobility. The reason is quite obvious: the most important clients of the network are moving vehicles. And vehicles may cover large distances in short periods of time. One of the most intuitive deployment strategies is to first cover the most dense location; that is, the location presenting the largest number of users is prioritized to receive the communication infrastructure. Such strategy is effective for cellular networks, but not for vehicular networks. Although placing RSUs at the densest locations seems reasonable, the assumption fails when we consider that those vehicles composing dense regions are originated from nearby and the dense region results of merging flows.

At first glance, it may seem reasonable to place works presenting deployment strategies in the same class as works presenting communication strategies. However, in this article we choose to keep them as separate classes, allowing us to present distinct discussions. Works assuming important premises in terms of the location of RSUs are considered to be part of the deployment class, while works addressing any aspect of the communication (routing, protocols, and data dissemination) without assuming premises regarding the location of RSUs are considered as pure communication works.

In fact, when we look at the evolution of works since early 2000s, we notice a clear distinction between works addressing communication aspects and works proposing deployment strategies. However, the gap between both classes is increasingly reduced over time. Basically, works originally addressing communication aspects incorporate more and more premises about the infrastructure supporting the operation of vehicular networks. With such premises becoming increasingly more assertive, it becomes hard to tell whether the work is addressing communication aspects or deployment aspects.

Since we performed this survey by reading each work individually in a time ascending order, such distinction is very clear in the early works, but it becomes fuzzy in more recent ones. Our perception indicates a tendency of works migrating from pure communication to deployment, which makes sense when we consider that the research community is moving towards infrastructure-based vehicular networks.

Deployment strategies are highly dependent on city dynamics and urban mobility. When we analyze real/realistic vehicular mobility traces (such as the Vehicular Mobility Trace of the City of Cologne (Germany) available at http://kolntrace.project.citi-lab.fr/), we notice flows of vehicles converging towards attraction (and very dense) areas. Furthermore, very dense regions do not appear as isolated islands, but they result from merging flows heading towards attraction areas.

Such issue indicates that very dense regions (in cities) tend to appear somehow interconnected, and vehicles traveling near very dense areas have high probability of joining the main flow. Thus, when we consider just the density of vehicles for placing the infrastructure, we may incur in redundant coverage by deploying RSUs covering the same flow several times, while vehicles traveling outside these very popular routes will not experience any vehicle-to-infrastructure contact.

In the remainder of this section, we overview selected works from the literature in order to present the evolution of this field. We identify the following classes of deployment strategies.

(i) Analytic Studies Addressing the Deployment of RSUs

(ii) Deployment Strategies Based on the V2I (Vehicle-toInfrastructure) Contact Probability

(iii) Deployment Strategies for the Distribution of Content

(iv) Deployment Strategies Based on Clustering

(v) Deployment Strategies Based on Geometry

(vi) Deployment Strategies Based on Evolutionary Approaches

(vii) Linear Programming Models for Solving the Deployment of RSUs

(viii) Deployment Strategies Based on the Maximum Coverage Problem.

5.1. Analytic Studies Addressing the Deployment of RSU. The state of the art for vehicular communication deals with simulations, field trials, and analytic studies. Simulations are scalable, inexpensive, and easy to perform and provide a full understanding before the real implementation, but they cannot represent exactly the real environments and happenings. Field trials are real but expensive, difficult to perform, and sometimes not easy to be interpreted. Analytic studies allow addressing specific issues (such as the impact of latency, the delivery rate, and the impact of the density of vehicles on performance) before implementing simulations and trials, with the great advantage of highlighting which parameter and conditions affect the performance with more importance. In the following, some relevant analytic studies are proposed, each one dealing with a different aspect of V2I communication.

Nekoui et al. [72] propose the definition of an infrastructure for vehicular networks based on the conventional definition of transport capacity. Authors develop a mathematical model where the destination nodes are chosen at random by the source nodes, and they study the impact of the infrastructure in the capacity of the vehicular networks. Using an analytic model, they show that exploiting any number of infrastructure nodes beyond a certain amount enhances the achievable capacity. Although the authors propose to handle arbitrary topologies, they assume several simplifications in the mobility model.

Alpha Coverage provides worst case guarantees on the interconnection gap [76]. A deployment of RSUs is considered $\alpha$-covered if any path of length $\alpha$ on the road network meets at least one RSU. The solution proposed by Alpha Coverage is very interesting, but it seems to make more sense 
when the network designer intends a massive deployment. As a critical analysis, in some sense, Alpha Coverage assumes the underlying premise that all roads share the same relevance and characteristics (speed, relevance to the city, density of traffic, etc.). However, when we consider real scenarios, roads tend to be very different in terms of several factors.

Sou and Tonguz [85] investigate the allocation of RSUs along a highway. They analyze the performance of RSUs taking into account important issues such as the vehicle deceleration, channel congestion, different beacon frequencies, hidden node problem, and multilane traffic. The results indicate that on a $300 \mathrm{~km}$ highway, the rehealing delay is reduced by $70 \%$ whereas the average number of rehealing hops is reduced by $68.4 \%$ when we deploy 50 RSUs when compared to an operation with no RSUs. Authors conclude that the deployment of a small number of RSUs can achieve a substantial improvement in sparse vehicular networks.

Bazzi et al. [97] address cellular systems as the most feasible solution in the short term to collect information messages from vehicles to a remote control center. The paper proposes a mathematical model to evaluate the impact of the envisioned service on cellular systems capacity and coverage in simplified scenarios. Results show that the acquisition of small and frequent packets from vehicles is affected by interference more than other services, such as the voice service.

Furthermore, analytical results highlight that this service could not be feasible where cells are planned for high coverage with low capacity, such as in interurban scenarios. Note, however, that, in such a scenario, the reduced number of roads and the limited alternatives do not motivate the acquisition of measurements from all vehicles with such strict delay constraints. In any case, advanced strategies could be investigated, such as the fragmentation of packets into smaller parts that can be transferred with a higher processing gain but with an increased occupation of the resources in the time domain, the storing of data until better coverage is not reached, implying a higher average delivery delay, the use of vehicle-to-vehicle communication to quickly collect higher amounts of measurements in one vehicle, and justifying the use of unicast transmissions.

\subsection{Deployment Strategies Based on the V2I Contact Proba-} bility. An important figure of merit in such a dynamic environment is the fraction of space and/or time of connectivity between the vehicle and the infrastructure, especially when the vehicle is out of coverage (of a cellular network eNodeB or of a RSU) and/or when the speed is high and the contact with a RSU is limited. The longer the contact opportunity, the easier the data transfer and the higher the quality of service.

The first work we could find in the literature is the one authored by $\mathrm{Li}$ et al. [178]. In this work, authors propose a deployment strategy similar to the base station placement in cellular systems. The goal is to minimize the power consumption and the average number of hops from access points to gateways under the assumption of full coverage by RSUs. Gateways connect access points to the Internet. Every vehicle is considered connected to access points. Vehicle speed, density, or movement patterns have not been considered.
Scheme does not take into account the interference problem and the road topology.

Zheng et al. [81] present the evaluation of a deployment strategy through the contact opportunity, which measures the fraction of distance or time that vehicles are in contact with the infrastructure. The authors also propose a deployment algorithm intended to maximize the worst case contact opportunity under budget constraints. The solution is evaluated using computer simulations and a testbed in a university campus. They consider two baseline algorithms: Random Deployment and Max-Min Distance Sampling. The Max-Min Distance Sampling [179] starts at a randomly selected location and allocates RSUs iteratively maximizing the minimum graph distance in terms of shortest paths.

Lee and Kim [79] propose a greedy heuristic to place RSUs aiming to improve the vehicles connectivity while reducing disconnections. The heuristic counts the amount of reached vehicles by each intersection considering the transmission range of RSUs. Each intersection is considered a potential location for receiving RSUs. The locations are selected based on the number of vehicles, and the heuristic does not take into account the speed or density of vehicles in a given area.

Chi et al. [107] propose three optimal algorithms to allocate RSUs: greedy, dynamic, and hybrid algorithms. Authors assume (i) placing RSUs preferentially at important intersections; (ii) allocating RSUs until every intersection is covered; and (iii) distributing RSUs as even as possible. The relevance of each intersection is evaluated using traffic factors including vehicles' density, intersection popularity, and intersection particularity. The greedy algorithm simply deploys RSUs at intersections in descending order of the intersection priority. The dynamic algorithm concentrates on the even distribution of RSUs, while the hybrid algorithm combines the previous ones, while Xiong et al. [180] propose Roadgate to address the placement of RSUs guaranteeing a probability of contact between vehicles and the infrastructure.

Bazzi et al. [181] discuss the system design and address the cellular offloading issue in urban scenarios through the deployment of WAVE/IEEE 802.11p devices on vehicles and RSUs. The work shows the impact of the percentage of equipped vehicles, of the number of deployed RSUs, and of the adopted routing protocols on the amount of data delivered. Results, obtained through an integrated simulation platform taking both realistic vehicular environments and wireless network communication aspects into account, show that the deployment of few roadside units and the use of low complexity routing protocols lead to a significant reduction of cellular resource occupation, even approaching 100\% with a high density of equipped vehicles. Hence, also the content distribution issue is, in part, addressed, as better explained in the following section.

5.3. Deployment Strategies for the Distribution of Content. There are also deployment strategies designed for the distribution of content. Data represent, in fact, a great richness and opportunity to develop new applications and service. Hence, the acquisition and distribution of data have acquired an 
increasing importance in recent years, especially for applications dealing with traffic management, user profiling, and environment monitoring. Trullols-Cruces et al. [182] introduce a mixed-integer quadratic programming based optimum RSUs' deployment scheme to provide Internet access services for the maximum road traffic volumes with limited number of RSUs.

Additionally, Liu et al. [24] propose a deployment strategy for file downloading in vehicular networks. The V2I encounters are modeled as a time continuous homogeneous Markov chain. Filippini et al. [92] investigate the scenario where two network providers are competing for market shares using games theory. They consider the distribution of content along a road of length $D$. Each RSU is characterized by a coverage range $R$, which defines its service area, and by an application level goodput for the content delivery. The goodput depends on the wireless technology of the RSU. Both simultaneous and leader-follower deployments are evaluated.

Silva et al. [131] propose the Sigma Deployment for modeling the distribution of several contents in vehicular networks in the style of a Mobile Content Delivery Vehicular Network. Since a given content may be meaningful only to a given region of interest, they assume that each content type is related to a target region where it must be made available. Furthermore, given the wide range of envisioned vehicular applications, each content requires distinct performance levels from the network defined in terms of vehicle-to-infrastructure contact probability and vehicle-toinfrastructure contact duration. A given layout of RSUs is considered a $\Sigma\left(\begin{array}{c}R_{c} \\ c\end{array}\right)\left(\begin{array}{c}\rho_{1} \\ \rho_{2}\end{array}\right)$ Deployment whenever $\rho_{2}$ percent of vehicles traveling $R_{c}$ are connected to RSUs able to provide the content $c$ during at least $\rho_{1}$ percent of the trip duration along $R_{c}$ ( $R_{c}$ is a subset of regions of the road network $R$, where the content $c$ has to be available). The parameters $\rho_{1}$ and $\rho_{2}$ indicate performance guarantees for vehicles finding the content $c$ inside the regions $R_{c}$ of the road network. Finally, Lu et al. [104] investigate the capacity-cost trade-offs in terms of the wireless access infrastructure.

5.4. Deployment Strategies Based on Clustering. Kchiche and Kamoun [80] propose a greedy algorithm based on the centrality of group to select the best locations for the infrastructure. The algorithm aims to maximize the performance of the message distribution system by reducing the global delay and the communication overhead of messages. The authors demonstrate that both the centrality and equidistance of the infrastructure is important to improve the quality of the coverage.

5.5. Deployment Strategies Based on Geometry. Cheng et al. [102] propose a geometry-based coverage strategy to handle the deployment problem over urban scenarios using the shape and area of road segments. Patil and Gokhale [103] propose a Voronoi [183] diagram-based algorithm for the deployment of infrastructure using the packet delay and packet loss as criteria. The authors provide a collaborative mechanism for dynamic resources management in vehicular networks, allowing managing the network quality of service. The collaboration between vehicles and RSUs is enabled through a vehicle-to-infrastructure network. They use population census within SUMO's ActivGen API to generate traffic data mimicking the real world.

Liya et al. [105] propose a randomized algorithm that calculates an approximate distance for deploying RSUs by approaching the optimal distance step by step from the initial distance $d_{0}=2 R_{0}$, where $R_{0}$ is the transmission range. The distance is sequentially increased to $d_{0}(1+\theta), d_{0}(1+$ $\theta)^{2}, \ldots, d_{0}(1+\theta)^{n}$, until the network cannot meet connectivity. As a critical analysis, the idea presented in the work seems to be very promising for massive deployments, and we can possibly improve the efficiency of the strategy by using a better strategy for assigning the RSUs.

Sou [78] addresses the placement of RSUs in rural areas and roadways where the solutions must deal with the low density of vehicles and larger areas to be covered. The author proposes the deployment of RSUs equally distanced from each other along a roadway enabling some RSUs to enter the power-saving mode optimizing energy consumption.

5.6. Deployment Strategies Based on Evolutionary Approaches. The use of virus-evolutionary genetic algorithms can be useful for real-time route planning and traffic forecasting. Lochert et al. [65] study how the infrastructure should be used to improve the travel time of data over large distances. The authors present a multilayer aggregation scheme defining landmarks. Cars passing landmarks record the time travel, which is aggregated to infer the time travel between more distant landmarks. These aggregation steps are performed by the cars themselves in a completely decentralized basis. The minimal initial deployment of RSUs is handled by a genetic algorithm based on the travel time savings. Cavalcante et al. [91] apply genetic programming to solve the deployment of RSUs in vehicular networks. Such technique starts with an initial set of possible solutions combined across generations until some stop condition is reached. The authors model the problem as a Maximum Coverage and they impose a time limit.

In Intelligent Transportation Systems, a key role is played by efficient route planning services. Such systems still have the lack of a full support of real-time traffic monitoring and the consequent real-time update of the best route suggested. In [184], an architecture for the management of dynamic path planning is proposed and limitations of traditional search algorithms in these kinds of applications are discussed. A variant of the proposed approach is consequently presented, based on the adoption of genetic algorithm to improve the efficiency of real-time navigation. The genetic approach considers many solutions at a time, so it acquires knowledge and improves the set of candidate solutions during the search process, improving the efficiency of global search of traditional algorithms. Further improvements are also proposed through virus-enhanced variant: whereas typical genetic algorithms may not be able to solve large-scale problems within a practical amount of time, viruses give a direction to the search, improving thus search rate and quality of solutions and speeding the whole process up. 
5.7. Linear Programming Models for Solving the Deployment of $R S U s$. Aslam et al. [87] use binary integer programming to solve the allocation of RSUs. They eliminate minor roads and model major roads as a grid. Authors present two different optimization methods for placement of a limited number of RSUs in an urban region: (a) analytical binary integer programming (BIP); (b) novel Balloon Expansion Heuristic (BEH). The BIP method utilizes branch and bound approach to find an optimal analytical solution whereas BEH method uses balloon expansion analogy to find an optimal or near optimal solution. Authors conclude that the BEH method is more versatile and performs better than BIP method in terms of the computational cost and scalability.

Wu et al. [89] focus on a highway scenario with multiple lanes, exits, and intersections along the road. Vehicles can communicate with the infrastructure or use multihop relay when out of the infrastructure's transmission range. Authors model the deployment of RSUs as an Integer Linear Program formulation considering both strategies of communication so that the aggregate throughput in the network is maximized. Authors also model the impact of wireless interference, vehicle population distribution, and vehicle speeds. The model is evaluated via ns-2 and VanetMobiSim (http://vanet.eurecom.fr/) simulations. Multihop relaying allows the vehicles to deliver packets forward to the RSUs ahead or backward, according to the smallest hop count. Authors demonstrate that the scheme overcomes uniformly distributed placement.

Liang et al. [88] study the deployment of the roadside infrastructure by formulating an optimization problem and solving it using Integer Linear Programming. The proposed optimization framework takes into account the effect of buildings on signal propagation, LAN lines, and road topology. The formulation assumes a grid-like road network.

Although using a grid-like road network may seem unrealistic, we can convert a complex road network into a grid-like structure by defining urban cells. We can partition the urban area into a set of $\psi \times \psi$ (same size) urban cells. The urban cells may have arbitrary sizes in order to meet the network designer needs. When we demand more/less accuracy, we simply increase/decrease the number of urban cells covering the road network.

Without loss of generality, we can assume that each urban cell is covered by deploying one single logical RSU. The logical RSU is the one able to entirely cover an urban cell. The concept of logical RSUs allows us to abstract from the exact location of RSUs inside urban cells, which seems to be more realistic since the exact physical deployment depends on taking into account several practical issues, such as the presence of energy supply, signal interference, presence of constructions blocking the signal, and others. The identification of such issues tends to demand in site inspection.

In other words, we split the deployment into two complementary subtasks. The intercell deployment is a highlevel selection of the regions (each region is an urban cell) that must be covered by logical RSUs. When solving the intercell deployment, we are not concerned on the exact location of the RSUs within the road network. We simply partition the road network and select the better urban cells for receiving coverage. Although the size of the urban cell can be made arbitrarily small, a good compromise seems to be achieved when the dimensions of the urban cell reflect the transmission range of RSUs. We call this intercell deployment. However, when considering the intracell deployment, we intend to define the exact set of devices that will be used for covering the selected urban cell. For instance, the network designer may choose between using a high transmission range device and using a few low-range devices, according to the specifics of the urban cell.

\subsection{Deployment Strategies Based on the Maximum Coverage} Problem. Trullols et al. [77] study the deployment of RSUs in urban areas. Authors use a realistic data set and propose modeling the deployment as a Knapsack Problem (KP) and also as a Maximum Coverage Problem (MCP-g). The heuristic MCP-g models the deployment of RSUs as the traditional Maximum Coverage Problem [165]. In fact, it is an adaption of the greedy solution for the Maximum Coverage Problem. In order to solve the MCP-g, we need previous and full knowledge of the trajectories of all vehicles. It receives as input a collection of sets, each set representing an intersection. The sets are defined over a domain of elements where each element represents a vehicle. MCP-g also receives a number $\alpha$ of available RSUs. The goal is to select at most $\alpha$ of these sets such that the union of the selected sets has maximal cardinality (i.e., select those intersections that cover the maximum number of uncovered vehicles). Authors demonstrate that MCP-g achieves close-to-optimum results. In the same study, the authors propose the KP heuristic that does not assume knowledge of the vehicles trajectories. The performance of KP is poor when compared to MCP-g.

Cataldi and Harri [90] propose the allocation of RSUs considering the Maximum Coverage Problem over a benefit function. The covered area is not a circle, but a polygonbased representing measured heterogeneous any-directional communication conditions. Authors argue that the coverage area of an infrastructure node cannot be modeled as a circular shape because the intensity may not reflect the quality of the experienced connectivity. The benefit function is nonhomogeneous over the covered area and the experiments use the simulator iTETRIS (An Integrated Wireless and Traffic Platform for Real-Time Road Traffic Management Solutions, http://www.ict-itetris.eu). Authors conclude that there exists an upper bound on the number of RSUs that needs to be allocated to some region.

Xie et al. [185] address the placement of RSUs in a gridlike road network assuming knowledge of the source and sink of each vehicle. Based on historical data, authors propose a probabilistic model to infer the best locations for RSUs. The probabilistic model also relies on a feature of the wireless link indicating the probability that a vehicle will get the information when driving through some RSU. This work is an extension of the proposal presented by [77]. Additionally, Yan et al. [106] propose a class of algorithms named Tailor to select a minimum number of intersections to deploy the infrastructure using a two-step approach.

Assuming previous knowledge of vehicles trajectories is a common strategy in deployment studies. However, when we 
TABLE 3: Comparison of deployment strategies.

\begin{tabular}{|c|c|}
\hline Deployment & Summary \\
\hline Analytic studies & $\begin{array}{l}\text { Theoretical studies addressing specific issues allowing us to better understand the } \\
\text { scenario before implementing simulations and trials. As a drawback, such } \\
\text { formulations may not represent exactly the real environments and happenings }\end{array}$ \\
\hline $\begin{array}{l}\text { Deployment Strategies } \\
\text { Based on the V2I Contact } \\
\text { Probability }\end{array}$ & $\begin{array}{l}\text { The V2I contact probability measures the expected number of contacts an average } \\
\text { vehicle tends to experiment during a typical trip. As a drawback, the V2I contact } \\
\text { probability is a generic measure that can help the deployment of simplistic } \\
\text { applications. However, as we increase the complexity of vehicular applications, we } \\
\text { demand a more complete set of measurements }\end{array}$ \\
\hline $\begin{array}{l}\text { Deployment Strategies for } \\
\text { the Distribution of Content }\end{array}$ & $\begin{array}{l}\text { Strategies for allocating RSUs in order to deliver large files, media, streaming, and } \\
\text { gaming }\end{array}$ \\
\hline $\begin{array}{l}\text { Deployment Strategies } \\
\text { Based on Clustering }\end{array}$ & $\begin{array}{l}\text { The use of clustering strategies may help the network designer to understand the } \\
\text { flow of vehicles and capture the most important zones in order to maximize the } \\
\text { coverage with a given set of resources }\end{array}$ \\
\hline $\begin{array}{l}\text { Geometry-Based } \\
\text { Deployment Strategies }\end{array}$ & $\begin{array}{l}\text { Such strategies rely on geometric properties of the city in order to define the most } \\
\text { promising locations for receiving RSUs. Such techniques are particularly promising } \\
\text { when combined with Geographical Information Systems and Georeferenced Data, } \\
\text { allowing full understanding of vehicular mobility. As a drawback, just a few authors } \\
\text { have exploited such strategy, and, as far as we are concerned, any author has applied } \\
\text { Geographical Information Systems for solving the deployment of RSUs }\end{array}$ \\
\hline $\begin{array}{l}\text { Deployment Strategies } \\
\text { Based on Evolutionary } \\
\text { Approaches }\end{array}$ & $\begin{array}{l}\text { Evolutionary approaches involve the use of metaheuristics inspired by the process } \\
\text { of natural selection commonly used to generate high-quality solutions to } \\
\text { optimization and search problems by relying on bioinspired operators such as } \\
\text { mutation, crossover, and selection }\end{array}$ \\
\hline Linear Programming & $\begin{array}{l}\text { Technique for the optimization of a linear objective function subject to linear } \\
\text { equality and linear inequality constraints. As a drawback, solving realistic scenarios } \\
\text { may be prohibitive given the required computational resources }\end{array}$ \\
\hline $\begin{array}{l}\text { Deployment Strategies } \\
\text { based on the Maximum } \\
\text { Coverage Problem (MCP) }\end{array}$ & $\begin{array}{l}\text { In MCP, we have a collection of sets, each set holding specific elements. The same } \\
\text { element can exist in multiple sets. The goal is to find the minimal collection of } k \\
\text { whose cardinality is maximal. Given its intrinsic nature, MCP is frequently used as } \\
\text { an abstraction for the deployment problem. As a drawback, MCP modeling tends to } \\
\text { have application as simplistic applications such as the dissemination of traffic } \\
\text { warnings. However, more complex applications demand more sophisticated } \\
\text { strategies for planning the roadside infrastructure }\end{array}$ \\
\hline
\end{tabular}

intend to maximize the number of distinct vehicles contacting the infrastructure, we may rely just on the migration ratios of vehicles between distinct locations of the road network. Since the migration ratios of vehicles do not require identifying individual vehicles, strategies based on the migration ratios do not incur in privacy concerns, while reducing the computational overhead for solving the deployment. Silva et al. [186] propose the use of turning ratios at each intersection of the road network as a basic information to infer the mobility of vehicles. Using turning ratios, the authors define the Probabilistic Maximum Coverage Problem (PMCP) as a model that improves the allocation of RSUs when we lack previous knowledge of the vehicles trajectories.

In a posterior work, Silva et al. [128] show how to generalize the application of turning ratios to large cities using the concept of urban cells. They divide the city into a grid-like structure and use the migrations ratios between adjacent urban cells. The infrastructure is deployed in urban cells selected according to the PMCP. Additionally, Silva et al. [129] propose the Full Projection of the Flow (FPF model) based on a Markovian Model to place the RSUs without the need to identify vehicles. The FPF model achieves close-tooptimal coverage when we intend to maximize the number of distinct vehicles contacting the infrastructure without relying on the trajectories information.

5.9. Comparative. In Table 3, we summarize the deployment strategies in terms of technique being applied.

5.10. Summary of Tools and Features for Studying the Deployment of Infrastructure for Vehicular Networks. On the basis of the overview of this section, we summarized the most relevant characteristics of the proposed deployment strategies, distinguishing various perspectives.

5.10.1. Optimization Targets. Given a fixed number of available RSUs, deployment strategies may have several optimization targets, some of which are summarized below:

(i) Maximum number of vehicle-to-infrastructure contacts

(ii) Maximum capacity of the network 
(iii) Minimizing the communication delay

(iv) Maximizing the connectivity of the network

(v) Maximizing data dissemination

(vi) Minimizing the energy consumption of the infrastructure

(vii) Minimizing the hop count of messages.

5.10.2. Deployment Scenarios. The main infrastructure deployment scenarios of interest are typically the following:

(i) Campus: often used in testbeds and realistic simulations

(ii) Highway: often used to represent sparse traffic

(iii) Random road networks: used in nonrealistic simulations

(iv) Rural: also used for sparse traffic

(v) Theoretical grid: easy to derive analytic expressions

(vi) Urban: often used in simulations.

From the perspective of location of RSUs, the scenarios can be further grouped into two main categories:

(i) Urban: majority of works consider that RSUs should be placed at intersections.

(ii) Highway/rural: studies about sparse traffic often rely on highways and rural areas. These efforts can be roughly characterized as equidistant and nonequidistant deployments.

5.10.3. Communication and Networking. Considering communication devices and network models, the works which appeared in the literature can be summarized as follows:

(i) Stationary RSUs

(ii) Publicly available Wi-Fi

(iii) Relays and meshes

(iv) Roadside buffers: devices used for storage and replication of messages

(v) Smart devices: devices offering some kind of feature to improve routing or data dissemination

(vi) Mobile RSUs (cars, buses, drones, and parked cars).

5.10.4. Vehicular Applications. The vehicular network deployment also depends on the data manipulated by the vehicular applications, which can be summarized as follows:

(i) File download: large files and streaming of video and music

(ii) Real-time data: interaction between drivers for gamming and voice

(iii) Traffic announcements: small self-contained messages.
5.10.5. Mobility Traces. The mobility traces used in the simulation of vehicular networks may be:

(i) real;

(ii) realistic;

(iii) synthetic (see the next subsection on mobility simulators);

(iv) generated using population census (via Sumo);

(v) unrealistic.

Examples of publicly available traces are the following:

(i) Geolife Trajectories (http://research.microsoft.com/enus/downloads/b16d359d-d164-469e-9fd4-daa38f2b2el3/);

(ii) San Francisco Cabs (http://cabspotting.org/);

(iii) Cologne (http://kolntrace.project.citi-lab.fr/);

(iv) Zurich (http://www.lst.inf.ethz.ch/research/ad-hoc/cartraces/);

(v) Beijing Taxi Traces (http://research.microsoft.com/enus/projects/tdrive);

(vi) Borlange (http://www.openstreetmap.org/traces?display_ name $=$ jetthe $\& \operatorname{tag}=$ Borl $\%$ C3\%A4nge);

(vii) Bologna (http://www.wcsg.ieiit.cnr.it/people/bazzi/ bazzi_projects.html).

5.10.6. Mobility Simulators. The most used mobility simulators are as follows:

(i) Corsim (http://mctrans.ce.ufl.edu/featured/tsis/)

(ii) SUMO (http://sumo-sim.org/)

(iii) Transims (https://code.google.com/p/transims/)

(iv) The One (DTN simulator) (http://www.netlab.tkk.fi/ tutkimus/dtn/theone/)

(v) VanetMobiSim (http://vanet.eurecom.fr/).

5.10.7. Network Simulators. The most used network simulators are as follows:

(i) Network Simulator 2 (http://www.isi.edu/nsnam/ns/)

(ii) Network Simulator 3 (http://www.nsnam.org/)

(iii) Omnet++ (http://www.omnetpp.org/)

(iv) Qualnet (http://web.scalable-networks.com/content/ qualnet/)

(v) iTETRIS (An Integrated Wireless and Traffic Platform for Real-Time Road Traffic Management Solutions) (http://www.ict-itetris.eu).

\section{Final Remarks and Future Directions}

In this article, we overviewed several works addressing infrastructure-based architectures, communications, and deployments for connected vehicles. Our goal was to summarize solutions proposed in the literature and to present 
an evolutionary picture of this research field. We organized the efforts into three categories: (i) deployment of infrastructures for vehicular networks; (ii) architectures for infrastructure-based vehicular networks; (iii) communication in infrastructure-based vehicular networks.

In a general sense, in the beginning of the 2000s, the works are concerned with identifying low level communication strategies allowing the vehicular communication. Most of the works deal with aspects of the vehicle-to-vehicle communication. Overtime, researchers become more interested in defining high-level aspects of the communication, such as providing Internet access to vehicles. The discussions on Intelligent Transportation Systems also drive the research towards the definition of novel applications.

The works start to make assumptions about a minimal infrastructure supporting the vehicular communication. Then, we have the first works proposing deployment strategies. Powered by more realistic mobility models and convinced of the need of a minimal infrastructure supporting the operation of vehicular networks, the research community gradually considers more assumptions about the placement of RSUs, and works that would originally deal only with communication or architectural aspects of the network, turn into deployment works.

In the years to come, the greatest challenge seems to be bringing Intelligent Transportation Systems into the streets. In order to achieve this, we demand the development of strategies for managing vehicular networks. Without them, governments will not be able to establish Service Level Agreements for network providers. The lack of strategies for managing vehicular networks also prevents network providers from planning future expansions of the network and computing the RoI (Return on Investments). Since Intelligent Transportation Systems are (typically) critical mission systems, and the decision-making is highly dependent on the data collected from the network, properly designing and managing the communication network is an essential step before deploying any vehicular application. Otherwise, there is reduced confidence on the availability and robustness of ITS systems.

Much more study is still to come. A thorough comprehension of the urban and rural mobility is certainly a crucial aspect for the development of ITS communication. An indepth understanding of urban and rural mobility enables us to design, develop, evaluate, and validate more realistic ITS models in terms of algorithms, analytic formulations, optimization models, and probabilistic approaches. More sophisticated ITS models support the development of better strategies for planning and managing the communication in ITS. Furthermore, better ITS models combined with a better understanding of the role played by mobile networks may even allow new insights into the application of the Internet of Things, cloud-based services, and Software Defined Networks (SDNs), allowing the development of more sophisticated services and applications. The management aspects of Software Defined Networks, Internet of Things, and clouds are crucially important in the development and wide deployment of these networks. Their adoption in ITS systems is also vitally important for their wider applicability and real deployment.

To provide more efficient and effective vehicular applications, the communication network management should enable easy establishment along roads and in low-density areas. Where network coverage cannot be guaranteed, deviceto-device (D2D) communication will be essential. 5G will represent a great technological breakthrough in this contest, by letting technologies and applications cooperate to achieve higher quality of service and experience. Furthermore, the use of social networking and crowd sourcing strategies may also represent a valuable source of management information for ITS. Handling large amounts of data from mobile devices in order to infer interesting knowledge and/or patterns may provide a turning point in terms of the ITS technology.

\section{Conflicts of Interest}

The authors declare that there are no conflicts of interest regarding the publication of this paper.

\section{References}

[1] D. F. Macedo, S. De Oliveira, F. A. Teixeira, A. L. L. Aquino, and R. A. Rabelo, "(CIA) 2-ITS: interconnecting mobile and ubiquitous devices for intelligent transportation systems," in Proceedings of the 2012 IEEE International Conference on Pervasive Computing and Communications Workshops, PERCOM Workshops 2012, pp. 447-450, March 2012.

[2] White Paper, European Transportation Policy for 2010: Time to Decide, Office for Official Publications of the European Communities, Luxembourg, 2001.

[3] H. Hartenstein and K. P. Laberteaux, "A tutorial survey on vehicular ad hoc networks," IEEE Communications Magazine, vol. 46, no. 6, pp. 164-171, 2008.

[4] S. Yousefi, M. S. Mousavi, and M. Fathy, "Vehicular Ad hoc Networks (VANETs): challenges and perspectives," in Proceedings of the 6th International Conference on ITS Telecommunications (ITST '06), pp. 761-766, June 2006.

[5] J. Jakubiak and Y. Koucheryavy, "State of the art and research challenges for VANETs," in Proceedings of the 5th IEEE Consumer Communications and Networking Conference (CCNC '08), pp. 912-916, Las Vegas, Nev, USA, January 2008.

[6] C. Bergnhem, S. Shladover, and E. Coelingh, "Overview of platooning systems," in Proceedings of the 19th ITS World Congress, Vienna, Austria, 2012.

[7] T. Taleb, A. Benslimane, and K. B. Letaief, "Toward an effective risk-conscious and collaborative vehicular collision avoidance system," IEEE Transactions on Vehicular Technology, vol. 59, no. 3, pp. 1474-1486, 2010.

[8] K. C. Dey, A. Rayamajhi, M. Chowdhury, P. Bhavsar, and J. Martin, "Vehicle-to-vehicle $(\mathrm{V} 2 \mathrm{~V})$ and vehicle-to-infrastructure (V2I) communication in a heterogeneous wireless network - Performance evaluation," Transportation Research Part C: Emerging Technologies, vol. 68, pp. 168-184, 2016.

[9] Audi cars now talk to stoplights in vegas. IEEE Spectrum, 2016.

[10] U. C. Kozat and L. Tassiulas, "Throughput capacity of random ad hoc networks with infrastructure support," in Proceedings of the 9th Annual International Conference on Mobile Computing And Networking, pp. 55-56, San Diego, CA, USA, September 2003. 
[11] M. Gerla, B. Zhou, Y.-Z. Lee, F. Soldo, U. Lee, and G. Marfia, "Vehicular grid communications: The role of the internet infrastructure," in Proceedings of the 2nd Annual International Workshop on Wireless Internet, WICON '06, August 2006.

[12] N. Banerjee, M. D. Corner, D. Towsley, and B. N. Levine, "Relays, base stations, and meshes," in Proceedings of the 14th ACM international conference, pp. 81-91, San Francisco, CA, USA, September 2008.

[13] A. B. Reis, S. Sargento, and O. K. Tonguz, "On the performance of sparse vehicular networks with road side units," in Proceedings of the 2011 IEEE 73rd Vehicular Technology Conference, VTC2011-Spring, pp. 1-5, May 2011.

[14] K. Mershad, H. Artail, and M. Gerla, "ROAMER: roadside Units as message routers in VANETs," Ad Hoc Networks, vol. 10, no. 3 , pp. 479-496, 2012.

[15] Y. Wu, Y. Zhu, and B. Li, "Infrastructure-assisted routing in vehicular networks," in Proceedings of the IEEE Conference on Computer Communications (INFOCOM '12), pp. 1485-1493, IEEE, Orlando, FL, USA, March 2012.

[16] 3GPP, Study on LTE support for Vehicle to Everything (V2X) services. TR22.885, 2016.

[17] 3GPP, Study on LTE-based V2X services, TR36.885, 2016.

[18] A. Bazzi, B. M. Masini, and A. Zanella, "Performance analysis of V2v beaconing using LTE in direct mode with full duplex radios," IEEE Wireless Communications Letters, vol. 40, no. 6, pp. 685-688, 2015.

[19] M. Centenaro, L. Vangelista, A. Zanella, and M. Zorzi, "Longrange communications in unlicensed bands: The rising stars in the IoT and smart city scenarios," IEEE Wireless Communications, vol. 230, no. 5, pp. 60-67, 2016.

[20] W. Viriyasitavat, F. Bai, and O. K. Tonguz, "UV-CAST: An urban vehicular broadcast protocol," in Proceedings of the 2010 IEEE Vehicular Networking Conference, VNC 2010, pp. 25-32, December 2010.

[21] V. P. Harigovindan, A. V. Babu, and L. Jacob, "Proportional fair resource allocation in vehicle-to-infrastructure networks for drive-thru Internet applications," Computer Communications, vol. 400, pp. 33-50, 2014.

[22] H. Liang and W. Zhuang, "Cooperative data dissemination via roadside WLANs," IEEE Communications Magazine, vol. 500, no. 4, pp. 68-74, 2012.

[23] F. Malandrino, C. Casetti, C.-F. Chiasserini, and M. Fiore, "Content downloading in vehicular networks: What really matters," in Proceedings of the IEEE INFOCOM 2011, pp. 426430, April 2011.

[24] Y. Liu, J. Niu, J. Ma, and W. Wang, "File downloading oriented Roadside Units deployment for vehicular networks," Journal of Systems Architecture, vol. 590, no. 10, Part B, pp. 938-946, 2013.

[25] C. Lochert, B. Scheuermann, M. Caliskan, and M. Mauve, “The feasibility of information dissemination in vehicular ad-hoc networks," in Proceedings of the 2007 Fourth Annual Conference on Wireless on Demand Network Systems and Services, WONS'07, pp. 92-99, January 2007.

[26] R. Bruno and M. Nurchis, "Robust and efficient data collection schemes for vehicular multimedia sensor Networks," in Proceedings of the 2013 IEEE 14th International Symposium on a World of Wireless, Mobile and Multimedia Networks, WoWMoM 2013, June 2013.

[27] H.-H. Chen, S. Ou, K. Yang, and A. Galis, "A selective downlink scheduling algorithm to enhance quality of VOD services for WAVE networks," Eurasip Journal on Wireless Communications and Networking, vol. 2009, Article ID 478157, 2009.
[28] C. E. Palazzi, M. Roccetti, S. Ferretti, and S. Frizzoli, "How to let gamers play in infrastructure-based vehicular networks," in Proceedings of the 2008 International Conference in Advances, pp. 95-98, New York, NY, USA, December 2008.

[29] C. E. Palazzi, S. Ferretti, and M. Roccetti, "Smart Access Points on the road for online gaming in vehicular networks," Entertainment Computing, vol. 10, no. 1, pp. 17-26, 2009.

[30] H. Wu, R. Fujimoto, M. Hunter, and R. Guensler, "An architecture study of infrastructure-based vehicular networks," in Proceedings of the 8th ACM international symposium, pp. 36-39, Quebec, Canada, October 2005.

[31] I. Ramani and S. Savage, "SyncScan: practical fast handoff for 802.11 infrastructure networks," in Proceedings of the Proceedings IEEE 24th Annual Joint Conference of the IEEE Computer and Communications Societies., pp. 675-684, Miami, FL, USA.

[32] V. Brik, A. Mishra, and S. Banerjee, "Eliminating handoff latencies in 802.11 WLANs using multiple radios," in Proceedings of the 5th ACM SIGCOMM Conference, p. 27, Berkeley, CA, USA, October 2005.

[33] Y. Amir, C. Danilov, M. Hilsdale, R. Musaloiu-Elefteri, and N. Rivera, "Fast handoff for seamless wireless mesh networks," in Proceedings of the 4th international conference, pp. 83-95, New York, NY, USA, June 2006.

[34] A. Boukerche, H. A. B. F. Oliveira, E. F. Nakamura, and A. A. F. Loureiro, "Vehicular Ad Hoc networks: a new challenge for localization-based systems," Computer Communications, vol. 310, no. 12, pp. 2838-2849, 2008.

[35] U. T. Rosi, C. S. Hyder, and T. Kim, "A Novel Approach for Infrastructure Deployment for VANET," in Proceedings of the 2008 Second International Conference on Future Generation Communication and Networking (FGCN), pp. 234-238, Hainan, China, December 2008.

[36] T. H. Luan, X. Ling, and X. Shen, "Provisioning QoS controlled media access in vehicular to infrastructure communications," Ad Hoc Networks, vol. 100, no. 2, pp. 231-242, 2012.

[37] C. M. Silva and W. Meira, "Evaluating the performance of heterogeneous vehicular networks," in Proceedings of the 82th IEEE Vehicular Technology Conference, VTC Fall 2015, September 2015.

[38] C. M. Silva, D. L. Guidoni, F. S. Souza et al., "Using the intercontact time for planning the communication infrastructure in vehicular networks," in Proceedings of the 2016 IEEE 19th International Conference on Intelligent Transportation Systems (ITSC), pp. 2089-2094, Rio de Janeiro, Brazil, November 2016.

[39] C. M. Silva, D. L. Guidoni, F. S. Souza, C. G. Pitangui, J. F. Sarubbi, and A. Pitsillides, "Gamma Deployment: Designing the Communication Infrastructure in Vehicular Networks Assuring Guarantees on the V2I Inter-Contact Time," in Proceedings of the 2016 IEEE 13th International Conference on Mobile Ad Hoc and Sensor Systems (MASS), pp. 263-271, Brasilia, Brazil, October 2016.

[40] G. Korkmaz, E. Ekici, and F. Özgüner, "Supporting realtime traffic in multihop vehicle-to-infrastructure networks," Transportation Research Part C: Emerging Technologies, vol. 180, no. 3, pp. 376-392, 2010.

[41] K. Liu and V. C. S. Lee, "RSU-based real-time data access in dynamic vehicular networks," in Proceedings of the 13th International IEEE Conference on Intelligent Transportation Systems, ITSC 2010, pp. 1051-1056, September 2010.

[42] A. Abdrabou, B. Liang, and W. Zhuang, "Delay analysis for a reliable message delivery in sparse vehicular ad hoc networks," 
in Proceedings of the 53th IEEE Global Communications Conference, GLOBECOM 2010, pp. 1-5, December 2010.

[43] D. Borsetti and J. Gozalvez, "Infrastructure-assisted geo-routing for cooperative vehicular networks," in Proceedings of the 2010 IEEE Vehicular Networking Conference, VNC 2010, pp. 255-262, December 2010.

[44] S. Annese, C. Casetti, C.-F. Chiasserini, N. Di Maio, A. Ghittino, and M. Reineri, "Seamless connectivity and routing in vehicular networks with infrastructure," IEEE Journal on Selected Areas in Communications, vol. 290, no. 3, pp. 501-514, 2011.

[45] F. Gómez Mármol and G. Martínez Pérez, "TRIP, a trust and reputation infrastructure-based proposal for vehicular ad hoc networks," Journal of Network and Computer Applications, vol. 350, no. 3, pp. 934-941, 2012.

[46] P. J. F. Ruiz, C. A. N. Guerra, and A. F. G. Skarmeta, "Deployment of a secure wireless infrastructure oriented to vehicular networks," in Proceedings of the 24th IEEE International Conference on Advanced Information Networking and Applications, AINA2010, pp. 1108-1114, April 2010.

[47] K. Plobl and H. Federrath, "A privacy aware and efficient security infrastructure for vehicular ad hoc networks," Computer Standards Interfaces, vol. 300, no. 6, pp. 390-397, 2008.

[48] T. R. Oliveira, C. M. Silva, D. F. Macedo, and J. M. Nogueira, "SNVC: Social networks for vehicular certification," Computer Networks, vol. 111, pp. 129-140, 2016.

[49] W. Zhang, Y. Chen, Y. Yang et al., "Multi-hop connectivity probability in infrastructure-based vehicular networks," IEEE Journal on Selected Areas in Communications, vol. 300, no. 4, pp. 740-747, 2012.

[50] M. Jerbi, S. M. Senouci, Y. G. Doudane, and A.-L. Beylot, "Geolocalized virtual infrastructure for urban vehicular networks," in Proceedings of the 2008 8th International Conference on Intelligent Transport System Telecommunications, ITST 2008, pp. 305-310, October 2008.

[51] J. Luo, X. Gu, T. Zhao, and W. Yan, "MI-VANET: A New Mobile Infrastructure Based VANET Architecture for Urban Environment," in Proceedings of the 2010 IEEE Vehicular Technology Conference (VTC 2010-Fall), pp. 1-5, Ontario, Canada, September 2010.

[52] T. Mishra, D. Garg, and M. M. Gore, "A Publish/Subscribe Communication Infrastructure for VANET Applications," in Proceedings of the 2011 IEEE Workshops of International Conference on Advanced Information Networking and Applications (WAINA), pp. 442-446, Biopolis, Singapore, March 2011.

[53] O. K. Tonguz and W. Viriyasitavat, "Cars as roadside units: A self-organizing network solution," IEEE Communications Magazine, vol. 51, no. 12, pp. 112-120, 2013.

[54] C. Sommer, D. Eckhoff, and F. Dressler, "IVC in cities: signal attenuation by buildings and how parked cars can improve the situation," IEEE Transactions on Mobile Computing, vol. 13, no. 8, pp. 1733-1745, 2014.

[55] C. M. Silva and W. Meira, "An architecture integrating stationary and mobile roadside units for providing communication on intelligent transportation systems," in Proceedings of the 2016 IEEE/IFIP Network Operations and Management Symposium, NOMS 2016, pp. 358-365, April 2016.

[56] T. H. Luan, L. X. Cai, J. Chen, X. S. Shen, and F. Bai, "Engineering a distributed infrastructure for large-scale cost-effective content dissemination over urban vehicular networks," IEEE Transactions on Vehicular Technology, vol. 63, no. 3, pp. 14191435, 2014.
[57] G. Marfia, G. Pau, E. De Sena, E. Giordano, and M. Gerla, "Evaluating vehicle network strategies for downtown Portland: Opportunistic infrastructure and the importance of realistic mobility models," in Proceedings of the 5th International Conference on Mobile Systems, Applications and Services, pp. 47-51, June 2007.

[58] Y. Zhang, J. Zhao, and G. Cao, "On scheduling vehicle-roadside data access," in Proceedings of the 4th ACM International Workshop on Vehicular Ad Hoc Networks (VANET '07), pp. 918, New York, NY, USA, September 2007.

[59] V. Taliwal, D. Jiang, H. Mangold, C. Chen, and R. Sengupta, "Empirical determination of channel characteristics for DSRC vehicle-to-vehicle communication," in Proceedings of the first ACM workshop, p. 88, Philadelphia, PA, USA, October 2004.

[60] B. Masini, C. Fontana, and R. Verdone, "Provision of an emergency warning service through GPRS: performance evaluation," in Proceedings of the 7th International IEEE Conference on Intelligent Transportation Systems, pp. 1098-1102, Washington, DC, USA.

[61] D. Hadaller, S. Keshav, and T. Brecht, "MV-MAX," in Proceedings of the 2006 SIGCOMM workshop, pp. 269-276, Pisa, Italy, September 2006.

[62] B. Masini, L. Zuliani, and O. Andrisano, "On the Effectiveness of a GPRS based Intelligent Transportation System in a Realistic Scenario," in Proceedings of the 2006 IEEE 63rd Vehicular Technology Conference, pp. 2997-3001, Melbourne, Australia.

[63] G. Korkmaz, E. Ekici, and F. Özgüner, "A cross-layer multihop data delivery protocol with fairness guarantees for vehicular networks," IEEE Transactions on Vehicular Technology, vol. 550, no. 3, pp. 865-875, 2006.

[64] A. Bazzi, B. M. Masini, A. Conti, and O. Andrisano, "Infomobility provision through MBMS/UMTS in realistic scenarios," in Proceedings of the 11th International IEEE Conference on Intelligent Transportation Systems, ITSC 2008, pp. 25-30, December 2008.

[65] C. Lochert, B. Scheuermann, C. Wewetzer, A. Luebke, and M. Mauve, "Data aggregation and roadside unit placement for a vanet traffic information system," in Proceedings of the 5th ACM International Workshop on VehiculAr Inter-NETworking (VANET '08), pp. 58-65, September 2008.

[66] J. Eriksson, L. Girod, B. Hull, R. Newton, S. Madden, and H. Balakrishnan, "The pothole patrol: using a mobile sensor network for road surface monitoring," in Proceedings of the 6th International Conference on Mobile Systems, Applications, and Services (MobiSys '08), pp. 29-39, Breckenridge, Colo, USA, June 2008.

[67] Waze mobile app. 2008, http://www.waze.com.

[68] J. Rybicki, B. Scheuermann, W. Kiess, C. Lochert, P. Fallahi, and M. Mauve, "Challenge: peers on wheels-a road to new traffic information systems," in Proceedings of the 13th Annual ACM International Conference on Mobile Computing and Networking (MobiCom '07), pp. 215-221, New York, NY, USA, 2007.

[69] S. Smaldone, L. Han, P. Shankar, and L. Iftode, "Roadspeak: Enabling voice chat on roadways using vehicular social networks," in Proceedings of the 1st Workshop on Social Network Systems, SocialNets'08, pp. 43-48, March 2008.

[70] M. Khabazian and M. K. Ali, "Generalized Performance Modeling of Vehicular Ad Hoc Networks (VANETs)," in Proceedings of the 2007 IEEE Symposium on Computers and Communications, pp. 51-56, Santiago, Portugal, July 2007.

[71] N. Wisitpongphan, F. Bai, P. Mudalige, and O. K. Tonguz, "On the routing problem in disconnected vehicular ad Hoc 
networks," in Proceedings of the IEEE INFOCOM 2007: 26th IEEE International Conference on Computer Communications, pp. 2291-2295, May 2007.

[72] M. Nekoui, A. Eslami, and H. Pishro-Nik, "The capacity of vehicular ad hoc networks with infrastructure," in Proceedings of the 6th Intl. Symposium on Modeling and Optimization in Mobile, Ad Hoc, and Wireless Networks, Wiopt 2008, pp. 267272, April 2008.

[73] A. Capone, M. Cesana, S. Napoli, and A. Pollastro, "MobiMESH: a Complete Solution for Wireless Mesh Networking," in Proceedings of the 2007 IEEE Internatonal Conference on Mobile Adhoc and Sensor Systems, pp. 1-3, Pisa, Italy, October 2007.

[74] J. Ormont, J. Walker, S. Banerjee, A. Sridharan, M. Seshadri, and S. Machiraju, "A city-wide vehicular infrastructure for widearea wireless experimentation," in Proceedings of the third ACM international workshop, pp. 3-10, San Francisco, CA, USA, September 2008.

[75] M. Fiore and J. Härri, "The networking shape of vehicular mobility," in Proceedings of the 9th ACM International Symposium on Mobile Ad Hoc Networking and Computing, MobiHoc '08, pp. 261-272, New York, NY, USA, May 2008.

[76] Z. Zheng, P. Sinha, and S. Kumar, "Alpha coverage: bounding the interconnection gap for vehicular internet access," in Proceedings of the IEEE INFOCOM, pp. 2831-2835, IEEE, April 2009.

[77] O. Trullols, M. Fiore, C. Casetti, C. Chiasserini, and J. Barcelo Ordinas, "Planning roadside infrastructure for information dissemination in intelligent transportation systems," Computer Communications, vol. 330, no. 4, pp. 432-442, 2010.

[78] S. Sou, "A Power-Saving Model for Roadside Unit Deployment in Vehicular Networks," IEEE Communications Letters, vol. 140, no. 7, pp. 623-625, 2010.

[79] J. Lee and C. Kim, "A roadside unit placement scheme for vehicular telematics networks," in Advances in Computer Science and Information Technology, T. Kim and H. Adel, Eds., vol. 6059 of Lecture Notes in Computer Science, 2016.

[80] A. Kchiche and F. Kamoun, "Centrality-based Access-Points deployment for vehicular networks," in Proceedings of the 2010 17th International Conference on Telecommunications, ICT 2010, pp. 700-706, April 2010.

[81] Z. Zheng, Z. Lu, P. Sinha, and S. Kumar, "Maximizing the contact opportunity for vehicular internet access," in Proceedings of the IEEE INFOCOM 2010, pp. 1-9, March 2010.

[82] A. Abdrabou and W. Zhuang, "On a stochastic delay bound for disrupted vehicle-to-infrastructure communication with random traffic," in Proceedings of the 2009 IEEE Global Telecommunications Conference, GLOBECOM 2009, December 2009.

[83] S. C. Ng and G. Mao, "Analysis of k-hop connectivity probability in 2-D wireless networks with infrastructure support," in Proceedings of the 53rd IEEE Global Communications Conference, GLOBECOM 2010, December 2010.

[84] S. C. Ng, W. Zhang, Y. Yang, and G. Mao, "Analysis of access and connectivity probabilities in infrastructure-based vehicular relay networks," in Proceedings of the IEEE Wireless Communications and Networking Conference 2010, WCNC 2010, April 2010.

[85] S.-I. Sou and O. K. Tonguz, "Enhancing VANET connectivity through roadside units on highways," IEEE Transactions on Vehicular Technology, vol. 60, no. 8, pp. 3586-3602, 2011.

[86] A. Abdrabou and W. Zhuang, "Probabilistic delay control and road side unit placement for vehicular ad hoc networks with disrupted connectivity," IEEE Journal on Selected Areas in Communications, vol. 29, no. 1, pp. 129-139, 2011.
[87] B. Aslam, F. Amjad, and C. C. Zou, "Optimal roadside units placement in urban areas for vehicular networks," in Proceedings of the 17th IEEE Symposium on Computers and Communication, ISCC 2012, pp. 000423-000429, July 2012.

[88] Y. Liang, H. Liu, and D. Rajan, "Optimal placement and configuration of roadside units in vehicular networks," in Proceedings of the IEEE 75th Vehicular Technology Conference, VTC Spring 2012, June 2012.

[89] T. Wu, W. Liao, and C. Chang, "A Cost-Effective Strategy for Road-Side Unit Placement in Vehicular Networks," IEEE Transactions on Communications, vol. 600, no. 8, pp. 2295-2303, 2012.

[90] P. Cataldi and J. Harri, "User/operator utility-based infrastructure deployment strategies for vehicular networks," in Proceedings of the IEEE 74th Vehicular Technology Conference, VTC Fall 2011, pp. 1-5, September 2011.

[91] E. S. Cavalcante, A. L. L. Aquino, G. L. Pappa, and A. A. F. Loureiro, "Roadside unit deployment for information dissemination in a VANET: an evolutionary approach," in Proceedings of the 14th Annual Conference Companion on Genetic and Evolutionary Computation (GECCO '12), pp. 27-34, ACM, Philadelphia, Pa, USA, July 2012.

[92] I. Filippini, F. Malandrino, G. Dán, M. Cesana, C. Casetti, and I. Marsh, "Non-cooperative RSU deployment in vehicular networks," in Proceedings of the 2012 9th Annual Conference on Wireless On-Demand Network Systems and Services, WONS 2012, pp. 79-82, January 2012.

[93] Z. Yu, J. Teng, X. Bai, D. Xuan, and W. Jia, "Connected coverage in wireless networks with directional antennas," in Proceedings of the IEEE INFOCOM 2011, pp. 2264-2272, April 2011.

[94] O. K. Tonguz, "Notice of violation of IEEE publication principles biologically inspired solutions to fundamental transportation problems," IEEE Communications Magazine, vol. 490, no. 11, pp. 106-115, 2011.

[95] S. Busanelli, G. Ferrari, V. A. Giorgio, and N. Iotti, "Information dissemination in urban vanets: single-hop or multihop?" Chapter contribution in Roadside Networks for Vehicular Communications: Architectures, Applications and Test Fields, pp. 237-263, 2012.

[96] T. Mangel and H. Hartenstein, "An analysis of data traffic in cellular networks caused by inter-vehicle communication at intersections," in Proceedings of the 2011 IEEE Intelligent Vehicles Symposium, IV'11, pp. 473-478, June 2011.

[97] A. Bazzi, B. M. Masini, and O. Andrisano, "On the frequent acquisition of small data through RACH in UMTS for its applications," IEEE Transactions on Vehicular Technology, vol. 600, no. 7, pp. 2914-2926, 2011.

[98] M. Koegel, W. Kiess, M. Kerper, and M. Mauve, "Compact vehicular trajectory encoding," in Proceedings of the 2011 IEEE 73th Vehicular Technology Conference, VTC2011-Spring, pp. 1-5, May 2011.

[99] S. Tayal and M. R. Triphath, "Vanet-challenges in selection of vehicular mobility model," in Proceedings of the 2012 Second International Conference on Advanced Computing \& Communication Technologies, pp. 231-235, Washington, DC, USA, 2012.

[100] S. Busanelli, G. Ferrari, and V. A. Giorgio, "I2V highway and urban vehicular networks: a comparative analysis of the impact of mobility on broadcast data dissemination," Journal of Communications, vol. 60, no. 1, pp. 87-100, 2011. 
[101] A. Bazzi, B. M. Masini, G. Pasolini, and O. Andrisano, "Smart navigation in intelligent transportation systems: Service performance and impact on wireless networks," IARIAInternationalJournalonAdvancesinTelecommunications, vol. 60, no. 7, pp. 57-70, 2013.

[102] H. Cheng, X. Fei, A. Boukerche, M. Abdelhamid, and M. Almulla, "A geometry-based coverage strategy over urban vanets," in Proceedings of the 10th ACM Symposium on Performance Evaluation of Wireless Ad Hoc, Sensor, \& Ubiquitous Networks PE-WASUN '13, pp. 121-128, New York, NY, USA.

[103] P. Patil and A. Gokhale, "Voronoi-based placement of road-side units to improve dynamic resource management in Vehicular Ad Hoc Networks," in Proceedings of the 2013 International Conference on Collaboration Technologies and Systems, CTS 2013, pp. 389-396, May 2013.

[104] N. Lu, N. Zhang, N. Cheng, X. Shen, J. W. Mark, and F. Bai, "Vehicles meet infrastructure: toward capacity-cost tradeoffs for vehicular access networks," IEEE Transactions on Intelligent Transportation Systems, vol. 140, no. 3, pp. 1266-1277, 2013.

[105] X. Liya, H. Chuanhe, L. Peng, and Z. Junyu, "A randomized algorithm for roadside units placement in vehicular ad hoc network," in Proceedings of the 9th IEEE International Conference on Mobile Ad-Hoc and Sensor Networks, MSN 2013, pp. 193-197, December 2013.

[106] T. Yan, W. Zhang, G. Wang, and Y. Zhang, "Access points planning in Urban area for data dissemination to drivers," IEEE Transactions on Vehicular Technology, vol. 630, no. 1, pp. 390402, 2014.

[107] J. Chi, Y. Jo, H. Park, and S. Park, "Intersection-priority based optimal RSU allocation for VANET," in Proceedings of the 5th International Conference on Ubiquitous and Future Networks, ICUFN 2013, pp. 350-355, July 2013.

[108] S. Busanelli, F. Rebecchi, M. Picone, N. Iotti, and G. Ferrari, "Cross-network information dissemination in vehicular ad hoc networks (VANETs): Experimental results from a smartphonebased testbed," Future Internet, vol. 50, no. 3, pp. 398-428, 2013.

[109] O. Kaiwartya, A. H. Abdullah, Y. Cao et al., "Internet of Vehicles: Motivation, Layered Architecture, Network Model, Challenges and Future Aspects," IEEE Access, vol. 4, no. 99, pp. 5356-5373, 2016.

[110] A. Fascista, G. Ciccarese, A. Coluccia, and G. Ricci, "A Localization Algorithm Based on V2I Communications and AOA Estimation," IEEE Signal Processing Letters, vol. 24, no. 1, pp. 126-130, 2017.

[111] B. W. Kim and S. Jung, "Vehicle Positioning Scheme Using V2V and V2I Visible Light Communications," in Proceedings of the 2016 IEEE 83th Vehicular Technology Conference (VTC Spring), pp. 1-5, Nanjing, China, May 2016.

[112] E. Okamoto, K. Kunitomo, H. Akita, and T. Kyo, "A Cooperative V2I Uplink Transmission Scheme Utilizing V2V Network Coding," in Proceedings of the 2016 IEEE 83th Vehicular Technology Conference (VTC Spring), pp. 1-5, Nanjing, China, May 2016.

[113] Afdhal and Elizar, "Enhanced route guidance and navigation for emergency vehicle using V2I-based cooperative communication," in Proceedings of the 17th International Electronics Symposium, IES 2015, pp. 145-150, September 2015.

[114] A. Ghosh, V. V. Paranthaman, G. Mapp, O. Gemikonakli, and J. Loo, "Enabling seamless V2I communications: Toward developing cooperative automotive applications in VANET systems," IEEE Communications Magazine, vol. 530, no. 12, pp. 80-86, 2015.
[115] K. Zheng, Q. Zheng, P. Chatzimisios, W. Xiang, and Y. Zhou, "Heterogeneous vehicular networking: a survey on architecture, challenges, and solutions," IEEE Communications Surveys and Tutorials, vol. 170, no. 4, pp. 2377-2396, 2015.

[116] A. Bazzi, B. M. Masini, A. Zanella, and A. Calisti, "Visible light communications in vehicular networks for cellular offloading," in Proceedings of the IEEE International Conference on Communication Workshop, ICCW 2015, pp. 1416-1421, June 2015.

[117] J. M. Marquez-Barja, H. Ahmadi, S. M. Tornell et al., "Breaking the vehicular wireless communications barriers: Vertical handover techniques for heterogeneous networks," IEEE Transactions on Vehicular Technology, vol. 640, no. 12, pp. 5878-5890, 2015.

[118] K. Zheng, Q. Zheng, H. Yang, L. Zhao, L. Hou, and P. Chatzimisios, "Reliable and efficient autonomous driving: the need for heterogeneous vehicular networks," IEEE Communications Magazine, vol. 530, no. 12, pp. 72-79, 2015.

[119] J. A. Sanguesa, F. Naranjo, V. Torres-Sanz, M. Fogue, P. Garrido, and F. J. Martinez, "On the study of vehicle density in intelligent transportation systems," Mobile Information Systems, vol. 2016, Article ID 8320756, 13 pages, 2016.

[120] B. M. Masini, "Vehicular networking for mobile crowd sensing," Ad Hoc Networks, vol. 36, pp. 407-408, 2016.

[121] A. Gorrieri, M. Martalò, S. Busanelli, and G. Ferrari, "Clustering and sensing with decentralized detection in vehicular ad hoc networks," Ad Hoc Networks, vol. 36, pp. 450-464, 2016.

[122] C.-C. Lo, K.-M. Chao, H.-Y. Kung, C.-H. Chen, and M. Chang, "Information Management and Applications of Intelligent Transportation System," Mathematical Problems in Engineering, vol. 2015, Article ID 613940, 2015.

[123] A. Gorrieri and G. Ferrari, "Irresponsible AODV routing," Vehicular Communications, vol. 20, no. 1, pp. 47-57, 2015.

[124] J. A. Sanguesa, M. Fogue, P. Garrido, F. J. Martinez, J.-C. Cano, and C. T. Calafate, "A survey and comparative study of broadcast warning message dissemination schemes for VANETs," Mobile Information Systems, vol. 2016, Article ID 8714142, 18 pages, 2016.

[125] M. Picone, M. Amoretti, G. Ferrari, and F. Zanichelli, "D4V: A peer-to-peer architecture for data dissemination in smartphone-based vehicular applications," PeerJ, vol. 2015, no. 1, article e15, 2015.

[126] A. Bazzi, A. Zanella, and B. M. Masini, "A distributed virtual traffic light algorithm exploiting short range V2V communications," Ad Hoc Networks, vol. 49, pp. 42-57, 2016.

[127] Z. Liu, M. Wu, K. Zhu, and L. Zhang, "SenSafe: A smartphonebased traffic safety framework by sensing vehicle and pedestrian behaviors," Mobile Information Systems, vol. 2016, Article ID 7967249, 2016.

[128] C. M. Silva, A. L.L. Aquino, and W. Meira, "Deployment of roadside units based on partial mobility information," Computer Communications, vol. 600, pp. 28-39, 2015.

[129] C. M. Silva, J. F. Sarubbi, and W. Meira, "Planning the communication infrastructure for vehicular networks without tracking vehicles," in Proceedings of the 2015 IEEE 11th International Conference on Wireless and Mobile Computing, Networking and Communications (WiMob), pp. 882-887, Abu Dhabi, United Arab Emirates, October 2015.

[130] C. M. Silva and W. Meira, "Design of roadside communication infrastructure with QoS guarantees," in Proceedings of the 2015 20th IEEE Symposium on Computers and Communication (ISCC), pp. 439-444, Larnaca, Cyprus, July 2015. 
[131] C. M. Silva, F. A. Silva, J. F. Sarubbi, T. R. Oliveira, W. Meira, and J. M. Nogueira, "Designing mobile content delivery networks for the internet of vehicles," Vehicular Communications, vol. 8, pp. 45-55, 2017.

[132] W. J. Franz, R. Eberhardt, and T. Luckenbach, "Fleetnet-internet on the road," in Proceedings of the 8th World Congresson Intelligent Transport Systems, 2001.

[133] V. Bychkovsky, K. Chen, M. Goraczko et al., "The cartel mobile sensor computing system," in Proceedings of the 4th international conference on Embedded networked sensor systems, SenSys, pp. 383-384, ACM.

[134] M. Gerla and L. Kleinrock, "Vehicular networks and the future of the mobile internet," Computer Networks, vol. 550, no. 2, pp. 457-469, 2011.

[135] P. E. Ross, Europe's smart highway, http://spectrum.ieee.org/ transportation/advanced-cars/europes-smart-highway-willshepherd-cars-from-rotterdam-to-vienna.

[136] Cooperative its corridor joint deployment, https://www.bmvi .de/SharedDocs/EN/Anlagen/VerkehrUndMobilitaet/Strasse/ cooperative-its-corridor.pdf?_blob=publicationFile.

[137] University of Michigan, connected Ann Arbor experiment web site, http://www.mtc.umich.edu/deployments/connected-ann-arbor.

[138] Tu-automotive japan 2016, http://www.tu-auto.com/japan/.

[139] Getting around in japan: The status and challenges of its, https:// www.fhwa.dot.gov/publications/publicroads/99marapr/japan.cfm.

[140] H. Wu, M. Palekar, R. Fujimoto et al., "An empirical stud y of short range communications for vehicles," in Proceedings of the 2nd ACM International Workshop on Vehicular Ad Hoc Networks (VANET '05), pp. 83-84, ACM, 2005.

[141] Vodafone demonstrates connected car over LTE at MWC, https:// www.telecompaper.com/background/vodafone-demonstratesconnected-car-over-lte-at-mwc-1186851.

[142] Consortium of automotive and telecom companies host 3GPP Cellular-V2X technology field trial in Germany, http://sites.ieee .org/connected-vehicles/2017/01/03/consortium-automotive-telecomcompanies-host-3gpp-cellular-v2x-technology-field-trial-germany/.

[143] Towards 5G initiative welcomes Qualcomm, shows Fast results, https://www.ericsson.com/news/170224-towards-5 g-initiativewelcomes-qualcomm_244010065_c.

[144] Vodafone, Bosch, Huawei test LTE-V2X in Germany, https:// www.telecompaper.com/news/vodafone-bosch-huawei-test-ltev2x-in-germany-1184329.

[145] UK CITE UK Connected Intelligent Transport Environment, https://www.ukcite.co.uk/.

[146] Deutsche Telekom trials LTE-V on the Ingolstadt autobahn test bed, http://www.telecomtv.com/articles/automotive/deutschetelekom-trials-lte-v-on-the-ingolstadt-autobahn-test-bed-13772/.

[147] Deutsche Telekom, Fraunhofer, Nokia and Continental run LTE V2X trials on Autobahn test bed, http://telematicswire.net/ deutsche-telekom-fraunhofer-nokia-and-continental-run-lte-v2xtrials-on-autobahn-test-bed/.

[148] I. F. Akyildiz, X. Wang, and W. Wang, "Wireless mesh networks: a survey," Computer Networks, vol. 470, no. 4, pp. 445-487, 2005.

[149] C. M. Silva, A. L. L. Aquino, and Meira W. Jr., "Smart traffic light for low traffic conditions," Mobile Networks and Applications, pp. 1-9, 2015.

[150] A. Bazzi, B. M. Masini, A. Zanella, and G. Pasolini, "IEEE 802.11p for cellular offloading in vehicular sensor networks," Computer Communications, vol. 60, pp. 97-108, 2015.
[151] S. Al-Sultan, M. M. Al-Doori, A. H. Al-Bayatti, and H. Zedan, "A comprehensive survey on vehicular Ad Hoc network," Journal of Network and Computer Applications, vol. 37, no. 1, pp. 380-392, 2014.

[152] A. Festag, "Standards for vehicular communicationfrom ieee 802.11p to $5 \mathrm{~g}$," eiElektrotechnik und Informationstechnik, vol. 1320, no. 7, pp. 409-416, 2015.

[153] C. Campolo, A. Molinaro, A. Vinel, N. Lyamin, and M. Jonsson, "Service discovery and access in vehicle-to-roadside multichannel VANETs," in Proceedings of the IEEE International Conference on Communication Workshop, ICCW 2015, pp. 24772482, June 2015.

[154] 3GPP. Service requirements for V2X services. TS 22.185, 2016.

[155] 3GPP. Evolved Universal Terrestrial Radio Access (E-UTRA); Physical channels and modulation. TS 36.211, 2016.

[156] 3GPP. Liaison statement from 3GPP RAN on LTE-based Vehicle-to-Vehicle communications. RP 161919, 2016.

[157] 3GPP. LTE-based V2X services. RP 161874, 2016.

[158] 3GPP. Study on enhancement of 3GPP support for 5G V2X services. TR 22.886, 2016.

[159] H. Cao, S. Gangakhedkar, A. R. Ali, M. Gharba, and J. Eichinger, "A 5G V2X testbed for cooperative automated driving," in Proceedings of the 2016 IEEE Vehicular Networking Conference (VNC), pp. 1-4, Columbus, OH, USA, December 2016.

[160] H. Droste, G. Zimmermann, M. Stamatelatos et al., "The METIS $5 \mathrm{G}$ architecture: a summary of METIS work on 5G architectures," in Proceedings of the 81th IEEE Vehicular Technology Conference (VTC '15), pp. 1-5, May 2015.

[161] J. Gozalvez, "Tentative 3GPP Timeline for 5G [Mobile Radio]," IEEE Vehicular Technology Magazine, vol. 100, no. 3, pp. 12-18, 2015.

[162] A. Nordrum, "Autonomous driving experts weigh 5G cellular network against dedicated short range communications," IEEE Spectrum, Cars That Think, May 2016.

[163] "IEEE standard for local and metropolitan area networks-part 15.7: short-range wireless optical communication using visible light," in IEEE Std, pp. 1-309, IEEE, 2011.

[164] J. A. Sanguesa, M. Fogue, P. Garrido, F. J. Martinez, J.-C. Cano, and C. T. Calafate, "A survey and comparative study of broadcast warning message dissemination schemes for VANETs," Mobile Information Systems, vol. 2016, Article ID 8714142, 18 pages, 2016.

[165] T. H. Cormen, C. E. Leiserson, R. Rivest, and C. Stein, Introduction to algorithmen, MIT press, 2001.

[166] Y. Zhang, J. Zhao, and G. Cao, "Service scheduling of vehicleroadside data access," Mobile Networks and Applications, vol. 150, no. 1, pp. 83-96, 2010.

[167] S. Panichpapiboon and G. Ferrari, "Irresponsible forwarding," in Proceedings of the 2008 8th International Conference on ITS Telecommunications (ITST), pp. 311-316, Phuket, Thailand, October 2008.

[168] M. Wang, Y. Zhang, C. Li, X. Wang, and L. Zhu, "A survey on intersection-based routing protocols in city scenario of VANETs," in Proceedings of the 2014 International Conference on Connected Vehicles and Expo (ICCVE), pp. 821-826, Vienna, Austria, November 2014.

[169] D. A. Johnson and M. M. Trivedi, "Driving style recognition using a smartphone as a sensor platform," in Proceedings of the 14th IEEE International Intelligent Transportation Systems Conference (ITSC '11), pp. 1609-1615, Washington, DC, USA, October 2011. 
[170] R. Araújo, Â. Igreja, R. De Castro, and R. E. Araújo, “Driving coach: a smartphone application to evaluate driving efficient patterns," in Proceedings of the 2012 IEEE Intelligent Vehicles Symposium, IV 2012, pp. 1005-1010, IEEE, Alcala de Henares, Spain, June 2012.

[171] M. F. Faraj, J. F. M. Sarubbi, C. M. Silva, M. F. Porto, and N. T. R. Nunes, "A real geographical application for the School Bus Routing Problem," in Proceedings of the 2014 17th IEEE International Conference on Intelligent Transportation Systems, ITSC 2014, pp. 2762-2767, IEEE, Qingdao, China, October 2014.

[172] C. M. Silva, J. F. M. Sarubbi, D. F. Silva, M. F. Porto, and N. T. R. Nunes, "A Mixed Load Solution for the Rural School Bus Routing Problem," in Proceedings of the 18th IEEE International Conference on Intelligent Transportation Systems, ITSC 2015, pp. 1940-1945, IEEE, Las Palmas, Spain, September 2015.

[173] E. Koukoumidis, L.-S. Peh, and M. R. Martonosi, "SignalGuru: leveraging mobile phones for collaborative traffic signal schedule advisory," in Proceedings of the 9th International Conference on Mobile Systems, Applications, and Services, pp. 127-140, ACM, July 2011.

[174] J. Zaldivar, C. T. Calafate, J. C. Cano, and P. Manzoni, “Providing accident detection in vehicular networks through OBD-II devices and android-based smartphones," in Proceedings of the 36th Annual IEEE Conference on Local Computer Networks (LCN '11), pp. 813-819, IEEE, Bonn, Germany, October 2011.

[175] C. Thompson, J. White, B. Dougherty, A. Albright, and D. C. Schmidt, "Using smartphones to detect car accidents and provide situational awareness to emergency responders," Lecture Notes of the Institute for Computer Sciences, Social-Informatics and Telecommunications Engineering, vol. 48, pp. 29-42, 2010.

[176] M. S. Al-Kahtani, "Survey on security attacks in vehicular ad hoc networks (VANETs)," in Proceedings of the 6th International Conference on Signal Processing and Communication Systems (ICSPCS '12), pp. 1-9, Queensland, Australia, December 2012.

[177] R. H. Frenkiel, B. R. Badrinath, J. Borràs, and R. D. Yates, "Infostations challenge: Balancing cost and ubiquity in delivering wireless data," IEEE Personal Communications, vol. 70, no. 2, pp. 66-71, 2000.

[178] P. Li, X. Huang, Y. Fang, and P. Lin, "Optimal placement of gateways in vehicular networks," IEEE Transactions on Vehicular Technology, vol. 560, no. 6, pp. 3421-3430, 2007.

[179] S. Teng, "Mutually Repellant Sampling," in Minimax and Applications, vol. 4 of Nonconvex Optimization and Its Applications, pp. 129-140, Springer, Boston, Mass, USA, 1995.

[180] Y. Xiong, J. Ma, W. Wang, and D. Tu, "RoadGate: mobilitycentric roadside units deployment for vehicular networks," International Journal of Distributed Sensor Networks, vol. 2013, Article ID 690974, 10 pages, 2013.

[181] A. Bazzi, B. M. Masini, A. Zanella, and G. Pasolini, "IEEE 802.11p for cellular offloading in vehicular sensor networks," Computer Communications, vol. 600, pp. 97-108, 2015.

[182] O. Trullols-Cruces, M. Fiore, and J. M. Barcelo-Ordinas, "Cooperative download in vehicular environments," IEEE Transactions on Mobile Computing, vol. 110, no. 4, pp. 663-678, 2012.

[183] F. Aurenhammer, "Voronoi diagrams-a survey of a fundamental geometric data structure," ACM Comput. Surv, vol. 230, no. 3, pp. 345-405, September 1991.

[184] C. De Castro, G. Leonardi, B. M. Masini, and P. Toppan, "An integrated architecture for infomobility services - advantages of genetic algorithms in real-time route planning," in Proceedings of the International Conference on Evolutionary Computation (ICEC), Oct 2010.
[185] B. Xie, G. Xia, Y. Chen, and M. Xu, "Roadside Infrastructure Placement for Information Dissemination in Urban ITS Based on a Probabilistic Model," in Network and Parallel Computing, vol. 8147 of Lecture Notes in Computer Science, pp. 322-331, Springer, Berlin, Heidelberg, 2013.

[186] C. M. Silva, A. L. L. Aquino, and W. Meira Jr., "Design of roadside infrastructure for information dissemination in vehicular networks," in Proceedings of the IEEE/IFIP Network Operations and Management Symposium: Management in a Software Defined World, NOMS 2014, pp. 1-8, May 2014. 

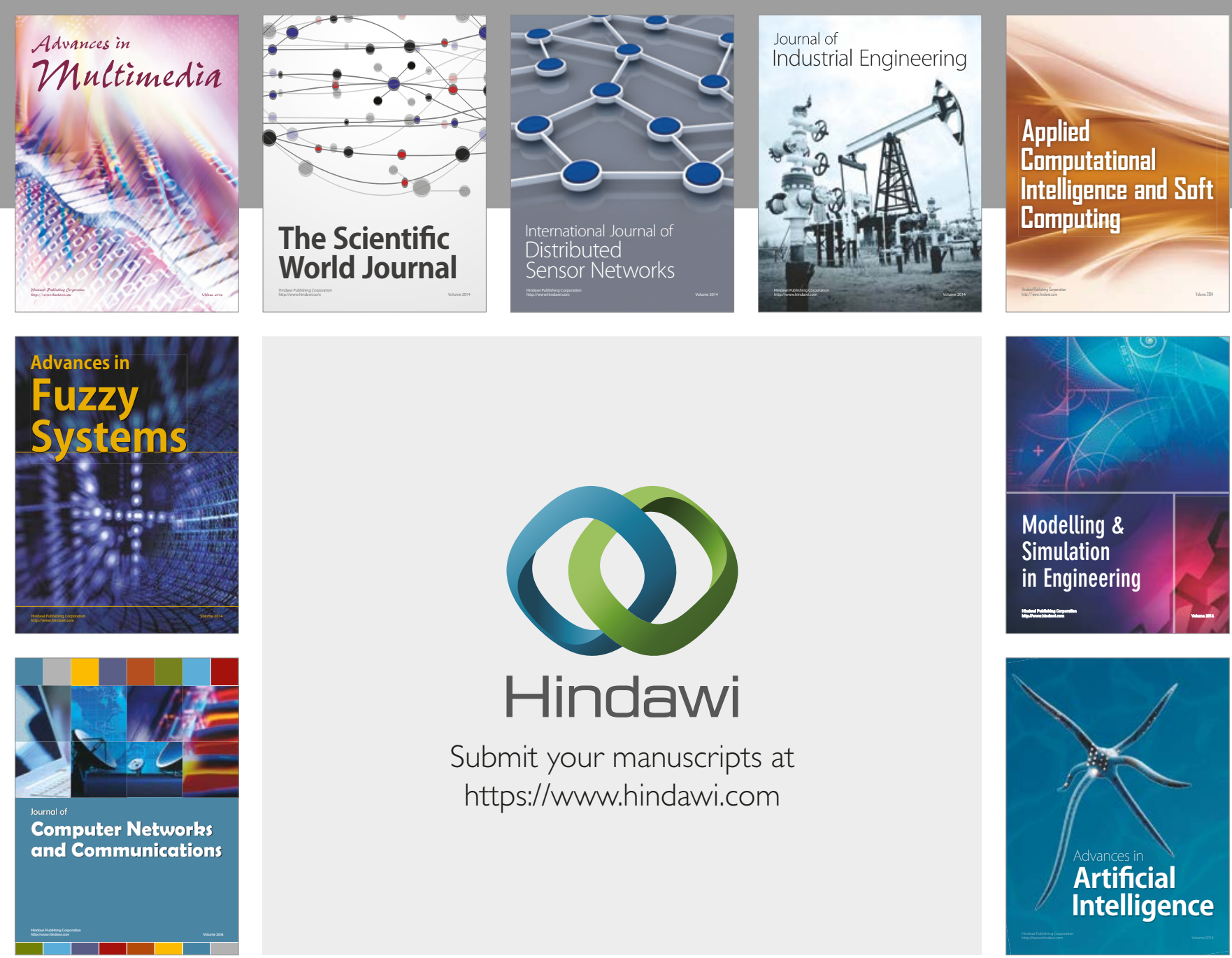

\section{Hindawi}

Submit your manuscripts at

https://www.hindawi.com
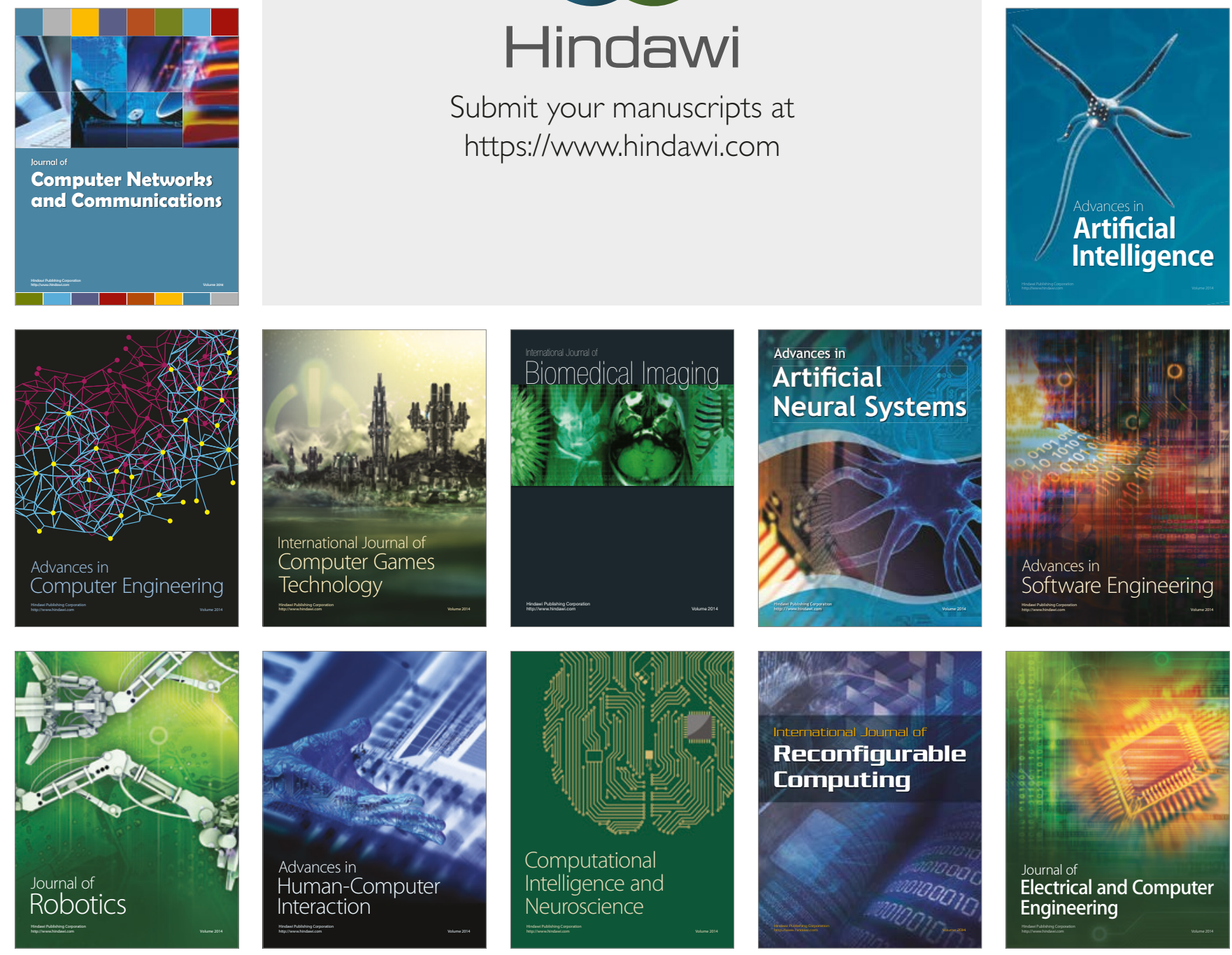